Stationary solutions and asymptotic flatness I

This content has been downloaded from IOPscience. Please scroll down to see the full text. 2014 Class. Quantum Grav. 31155012

(http://iopscience.iop.org/0264-9381/31/15/155012)

View the table of contents for this issue, or go to the journal homepage for more

Download details:

IP Address: 194.94.224.254

This content was downloaded on 09/09/2014 at 11:04

Please note that terms and conditions apply. 


\title{
Stationary solutions and asymptotic flatness I
}

\author{
Martin Reiris \\ Max Planck Institute für Gravitationsphysik, Golm, Germany \\ E-mail: martin@aei.mpg.de
}

Received 4 October 2013, revised 20 May 2014

Accepted for publication 23 May 2014

Published 22 July 2014

\begin{abstract}
In general relativity, a stationary isolated system is defined as an asymptotically flat (AF) stationary spacetime with compact material sources. Other definitions that are less restrictive on the type of asymptotic could in principle be possible. Between this article and its sequel, we show that under basic assumptions, asymptotic flatness indeed follows as a consequence of Einstein's theory. In particular, it is proved that any vacuum stationary spacetimeend whose (quotient) manifold is diffeomorphic to $\mathbb{R}^{3}$ minus a ball and whose Killing field has its norm bounded away from zero, is necessarily AF with Schwarzschildian fall off. The 'excised' ball would contain (if any) the actual material body, but this information is unnecessary to reach the conclusion. In this first article, we work with weakly asymptotically flat (WAF) stationary ends, a notion that generalizes as much as possible that of the AF end, and prove that WAF ends are AF with Schwarzschildian fall off. Physical and mathematical implications are also discussed.
\end{abstract}

Keywords: mathematical general relativity, stationary solutions, asymptotic flatness

PACS numbers: 02.40.-k, 04.20.-q.

\section{Introduction}

\subsection{A physical motivation}

Let us start with a peripheral discussion of some of the physical problems that motivated this research and which have to do with the asymptotics of the exterior regions of galaxies. We begin then with a succinct (but deliberately inaccurate) story about galaxies.

Around 13 billion years ago, the first galaxies started to form. Since then they accreted matter, delimited their visible shapes and, as the universe expanded, they drifted apart. As this happened, the profiles of their gravitational potentials settled and became a distinguishable 


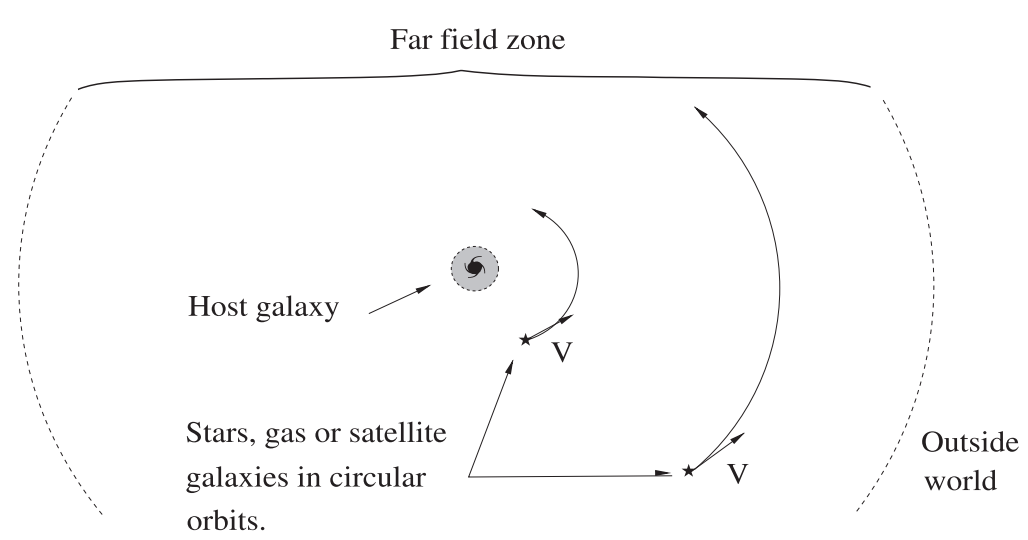

Figure 1. Representation of the far field zone of a disc galaxy (assuming dark matter halos were non-existent). Observationally, the velocity $|V|$ of test particles in circular orbits is fairly independent on the radius of the orbit (for a detailed account of the precise profiles of rotation curves, see $[7,10])$.

imprint of their material contents. One can thus imagine (see figure 1) that the space surrounding a given galaxy separates naturally into a bulk, a far field zone and, farther away, the outside world [5]. Moreover (it can be argued), the outside world, where all the other galaxies lie, is so far away that the far field zone can be modeled simply as an empty, asymptotically flat (AF), and stationary region of space, where only the potentials of the enclosed galaxy manifest.

This short and oversimplified picture sticks to some astronomical observations but not to all of them. Indeed, it is well established (see Sofue and Rubin [11] and, more recently, Salucci et al [7, 10]), that outside the visible regions of most disc galaxies ${ }^{1}$, the rotational velocity of gas, stars and satellite galaxies in circular orbits around the center of the galaxy is fairly independent on the radius of the orbit. A typical disc galaxy like the Milky Way can have, for instance, rotational velocities of the order of $220 \mathrm{~km} \mathrm{~s}^{-1}$. This means that gas, stars or even satellite galaxies orbiting at a certain distance from the visible edge of the host galaxy would revolve with a velocity of nearly $220 \mathrm{~km} \mathrm{~s}^{-1}$, and this will keep so for radii many times bigger than the visible radius of the galaxy (after which it will start to decrease [7, 10]). For the Milky Way, Zaritsky [15] reported that a flat rotation curve (as it is called) extends at least until $200 \mathrm{kpc}$, that is, more than 13 times the visible radius of the galaxy. The large and well known problem with all this is that, if we assume that the far field zone of galaxies is essentially empty, then the flat rotation curves cannot be explained solely by the gravitational attraction of the visible matter, at least within Newtonian theory. If this were the case then the rotational velocity should decrease inversely proportional to the square root of the radius, which, as we said, does not occur. The conventional and most plausible explanation to this peculiar phenomenon claims the existence of huge halos composed of weakly interacting massive particles enclosing the galaxies and causing such (dominating) gravitational distortions. If this is the case, the galaxies (understood here as only their visible parts), would not be isolated but rather immersed inside such huge material halos. If on the contrary halos do not exist, then galaxies could be probably thought of as isolated systems and the origin of such

${ }^{1}$ Elliptic galaxies and other systems also display flat rotation curves, but disc galaxies seem to be the most studied in this respect. 
big gravitational distortions in the far field zone demands an explanation. It is unlikely that in this last scenario an answer can be found within Newtonian theory only.

Based on these elements, it seems that an investigation into what general relativity (GR) can say in general about the gravitational field far from a compact source is rather justified. Roughly speaking, the aim is to study the type of asymptotics that non-compact stationary spacetimes with compact material sources can have. The less assumptions one makes on the geometry at infinity, the larger the applicability of the results. For this reason, the asymptotics of the spacetimes are considered in this investigation essentially as a geometric variable to be understood. In this sense one could be dealing with non-AF spacetimes for which the dynamics of test particles far away from the sources differ significantly from those of an AFspacetime. As we will see, this will not happen. We will discuss this in the next section. A physical incursion relevant to galaxies is given at the end of it.

To end, let us mention that the one above is just one among many other possible motivations for this work. The importance of studying isolated systems largely exceeds the sole analysis of galaxies.

\subsection{Overview of the main result}

In the Newtonian theory of gravity, an isolated system is simply one whose sources lie within a finite region of space (at every time). In the same fashion, it is customary to define isolated systems in GR by demanding asymptotic flatness (see for instance Ehlers [6]) and requiring that the material sources remain within a compact region of space (at every time). This choice however fixes the asymptotics a priori and is not of interest to us. Even for stationary spacetimes (those of consideration here), an adequate definition that does not fix the asymptotics of the spacetime a priori is not as clear as in Newtonian theory and requires some justification. In the following paragraphs we describe examples of stationary spacetimes and their asymptotics. Some of them will guide us to give a suitable redefinition of isolated systems in GR (definition 1.2). A discussion of the main result is given afterwards.

We begin with a simple spherically symmetric spacetime (with a singularity at the origin), displaying flat rotation curves similar to those showing up in galactic halos. For this reason this example is relevant in galactic dynamics (see remarks below). This spacetime (which is filled with matter), will not be particularly helpful to set our new definition of isolated system but is useful to contrast the radically different properties of vacuum and nonvacuum spacetime asymptotics, thus making a good conection with the previous section. It also shows very well the influence of the asymptotic itself in the dynamics of test particles. The example is a perfect-fluid solution of the Einstein equations which can be described explicitly as follows. The metric (in geometrized units) is given by

$$
\mathbf{g}=-r^{2 v^{2}} d t^{2}+\left(1+4 \pi v^{2}\left(2-v^{2}\right)\right) d r^{2}+r^{2} d \Omega^{2}
$$

where, as a simple calculation shows, the constant $v$ is the rotational velocity of circular orbits around the origin $r=0$ (hence constant). The stress-energy tensor is, as said, that of a perfect fluid with $\rho=\rho_{0} / r^{2}$ and $p=p_{0} / r^{2}$ where

$$
\rho_{0}=\frac{1}{8 \pi+2 /\left(\left(2-v^{2}\right) v^{2}\right)}, \quad \text { and } \quad p_{0}=\frac{v^{4}}{2+8 \pi\left(2-v^{2}\right) v^{2}} \text {. }
$$

This spacetime is found by solving the Tolman-Oppenheimer-Volkoff equation of hydrostatic equilibrium (see [14]) with the ansatz $\rho=\rho_{0} / r^{2}$ and $p=p_{0} / r^{2}$ and has a 


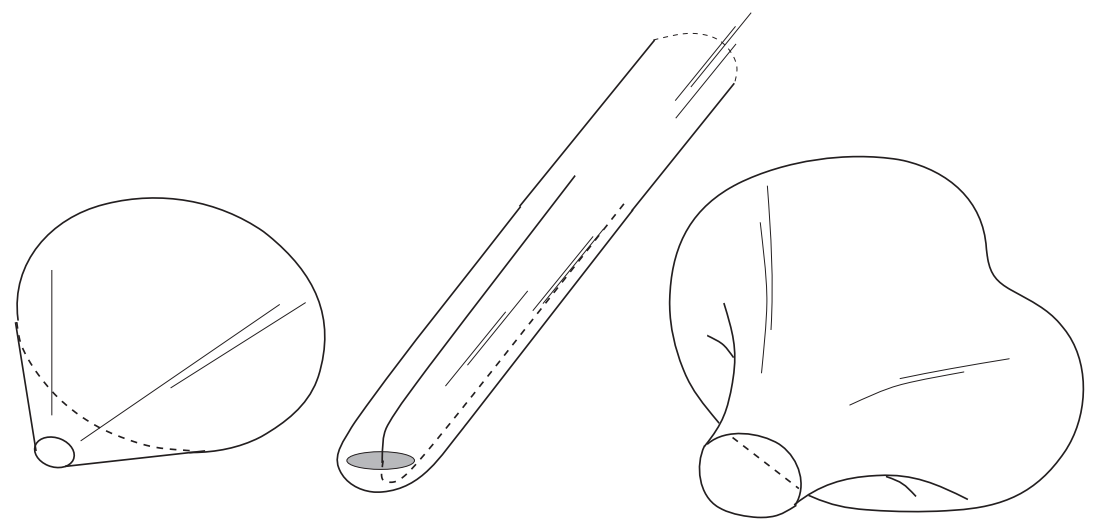

Figure 2. Representation of the space-like sections of the three different asymptotics discussed in the introduction. On the left is a self similar three-geometry. Centered is a rotating disc with the cylindrical asymptotics of a near-horizon geometry. On the right is an asymptotically flat three-geometry.

number of interesting properties. The Killing field $\partial_{t}$ is static and $r \partial_{r}+\left(1-v^{2}\right) t \partial_{t}$ is a conformal Killing field. Moreover $r \partial_{r}$ is a homothetic Killing field of the slice $t=0$ which is hence self similar and not AF (see figure 2). When $v \sim 0$ then $v \sim \sqrt{m(r) / r}$ where $m(r)$ is the mass contained until the coordinate radius $r$ (on the $t=0$ slice). Moreover the energy density $\rho_{0}$ measures the area defect as we have $8 \pi \rho_{0}=\left(A_{E}(\bar{r})-A(\bar{r})\right) / A_{E}(\bar{r})$ where here $A_{E}(\bar{r})=4 \pi \bar{r}^{2}$ is the Euclidean area of a sphere of radius $\bar{r}$ and $A(\bar{r})$ is the area of the sphere of metric radius $\bar{r}$ on the $t=0$ slice (i.e. $\bar{r}=\bar{r}(r)$ is the physical distance from the sphere of coordinate radius $r$ to the origin $r=0$ ). When $v \sim 0$ then (in natural units) $(v / c)^{2} \sim\left(A_{E}(\bar{r})-A(\bar{r})\right) / 2 A_{E}(\bar{r})$. In particular, for rotational velocities of the order of $300 \mathrm{~km} \mathrm{~s}^{-1}$ the area defect is of the order of $2 \times 10^{-6}$. The area defect (which does not depend on the radius), is thus a measure of the non-asymptotic flatness. It is worth noting that in the regime of small rotational velocities the spacetime just described reduces to the so called singular isothermal model that is widely used in galactic dynamics [13].

The previous is an interesting and relatively appealing stationary spacetime filled with matter and not AF. But what about stationary spacetimes with compact material sources? The paradigmatic case is of course the Schwarzschild solution which can represent for instance the gravitational field of a spherically symmetric star and which is AF. The Kerr black-holes (including Schwarzschild), can also be thought to represent compact bodies (the black-holes themselves) and there are other exact solutions, like the Tomimatsu-Sato class, that could also account for the exterior of isolated bodies. Interesting numerical solutions of stationary spacetimes with compact sources, also AF, have been studied extensively in the literature (see for instance [5] and references therein).

But, are there compact bodies or sources 'generating' a non-asymptotically flat stationary spacetime? Interestingly the answer is yes. In a series of papers (see [5] and references therein), Mainel and Neugebauer have shown that when a disc of dust rotates at a critical rate it 'generates' a stationary spacetime that is not AF but rather asymptotically cylindrical. More precisely far away from the disc the spacetime approaches the so called near horizon solution which has an appropriate space-like section displaying a cylindrical geometry (see figure 2). 
Concretely, such a slice of the near horizon geometry is diffeomorphic to $S^{2} \times \mathbb{R}$ and the initial data on it (normalized to have Komar angular momentum equal to one), has metric $g$ and second fundamental form $K$ given by

$$
\left\{\begin{array}{l}
g=\frac{4 \sin ^{2} \theta}{1+\cos ^{2} \theta} d \varphi^{2}+\left(1+\cos ^{2} \theta\right) d \theta^{2}+\left(1+\cos ^{2} \theta\right) d r^{2}, \\
K=\frac{2 \sin ^{2} \theta}{\left(1+\cos ^{2} \theta\right)^{\frac{3}{2}}}(d \varphi d r+d r d \varphi),
\end{array}\right.
$$

where $(\theta, \varphi)$ are the coordinates in $S^{2}$ and $r$ is the coordinate in the $\mathbb{R}$-factor. The metric has a translational symmetry along $r$ and is therefore cylindrical. The spacetime metric of its globally hyperbolic development is

$$
\begin{aligned}
\mathbf{g}= & -\left(1+\cos ^{2} \theta\right) d t^{2}+\left(1+\cos ^{2} \theta\right)(r \tan t d t+d r)^{2} \\
& +\frac{4 \sin ^{2} \theta}{\left(1+\cos ^{2} \theta\right)}(r d t-\varphi)^{2}+\left(1+\cos ^{2} \theta\right)^{2} d \theta^{2}
\end{aligned}
$$

which contains a Cauchy horizon at $t=\pi / 2$ and is not geodesically complete. This means that, no matter how far away we are from the disc of the critical Mainel-Neugebauer solution, there are always geodesics reaching a physical limit of the spacetime in a short parametric time. We will interpret this property as an indication that this spacetime should not be viewed as isolated (but this can be debatable of course). The definition 1.2 of the isolated system will incorporate this interpretation.

The critical Mainel-Neugebauer solution also has the peculiar property that its ergoregion (i.e. the region where the stationary Killing field is space-like), extends to infinity. The end of this spacetime is therefore not strictly stationary. This means that one cannot find a set of stationary observers covering the spacetime away from the sources. We will not contemplate such situations either in definition 1.2.

The previous discussion leads us essentially to only one possible definition. Very informally it says that a stationary isolated system is a stationary spacetime whose ends (denoted generically by $\mathbf{M}$ below), have the following three 'soft attributes'.

(1) The quotient of $\mathbf{M}$ by the stationary Killing field is diffeomorphic to $\mathbb{R}^{3}$ minus a ball.

(2) $\mathbf{M}$ is geodesically complete until the boundary, namely, geodesics either end at $\partial \mathbf{M}$ or are defined for infinite parametric time.

(3) The stationary Killing field has complete orbits and its norm is bounded away from zero.

The Schwarzschild ends (i.e. the domains $\mathbf{M}=\left\{(t, r, \theta, \varphi): r>r_{0}>2 m\right\}$ of the Schwarzschild spacetime), obviously enjoy 1, 2 and 3 above. Ends having the attributes 1, 2 and 3 will be called strongly stationary. The formal definition is as follows.

Definition 1.1. Let $(\mathbf{M}, \mathbf{g})$ be a smooth chronological solution of the vacuum Einstein equations with smooth boundary. Then, $(\mathbf{M}, \mathbf{g})$ is said to be a stationary spacetime end if the following conditions are fulfilled.

(S1) There is a time-like complete Killing field $X$ in $\mathbf{M}$ tangent to $\partial \mathbf{M}$ at $\partial \mathbf{M}$ such that the quotient of $\mathbf{M}$ by the orbits of $X$ is diffeomorphic to $\mathbb{R}^{3}$ minus an open ball. 
(S2) $(\mathbf{M}, \mathbf{g})$ is geodesically complete until the boundary, namely, geodesics either end at $\partial \mathbf{M}$ or are defined for infinite parametric time.

A stationary spacetime end $(\mathbf{M}, \mathbf{g})$ is said to be strongly stationary if in addition

(S3) There is a positive constant $c$ such that $-\langle X, X\rangle_{\mathbf{g}} \geqslant c$ all over $\mathbf{M},\left(\langle,\rangle_{\mathbf{g}}\right.$ is the g-inner product).

With this at hand the definition of the isolated system that we will adopt is the following.

Definition 1.2 (isolated system). A globally hyperbolic spacetime is called an isolated system if it is a union of, (i) finitely many strongly stationary ends and, (ii) an open region whose intersection with any Cauchy hypersurface has compact closure.

Note that, if any, the material sources would lie in the open region.

The main result that emerges between these two articles asserts that the ends of an isolated system (i.e. strongly stationary ends) are necessarily AF with Schwarzschildian fall off. Asymptotic flatness is, under soft assumptions, inevitable. The main result is therefore

Theorem 1.3 (main result). Isolated systems are AF with Schwarzschildian fall off.

Note that, because of the Scharzschildian fall off, the gravitational field at infinity of any isolated system coincides (loosely speaking) with that of a point-like source in Newtonian theory. In our view this result shows the remarkable internal structure of Einstein's theory.

The theorem 1.3 actually follows as a corollary of theorem 1.5, which is the main theorem to be proved in this series of articles. Theorem 1.5, that is stated in the next section, says that strongly stationary ends having cubic volume growth are AF with Schwarzschildian fall off. The notion of cubic volume growth as well as the deduction of theorem 1.3 from theorem 1.5 is also given in the next section.

The proof of the main theorem will be broken between the two articles of the series. In this first article we work with weakly asymptotically flat (WAF) stationary ends and prove that WAF ends are AF with Schwarzschildian fall off. The notion of the WAF end is a generalization of the notion of the weakly decaying stationary solution used by Kennefick and Murchadha in the seminal work [4], where they demonstrate (similarly to us here) that the Schwarzschildian fall off is the natural decay at infinity for stationary solutions. Roughly speaking, with WAF ends we are defining as much as could be allowed a notion of 'decaying into flat' without relying on any coordinate system and power-law decay for the curvature. Because of its generality, a rigorous definition of the WAF end requires some non-standard background material and therefore is postponed until section 3. Incidentally, by proving that WAF ends are AF we are answering a question raised in remark 2 of [4] which (quoted) says: 'the $\delta<0$ condition classically means that we are willing to consider any metric which decays to flat space as $1 / r^{\varepsilon}$ for any $\varepsilon$. Obviously one would like to replace this with 'going flat' and not require any kind of power law decay. It is difficult to see how this might be achieved; none of the present battery of weighted spaces (classical, Holder, .., ) seem suitable, ${ }^{2}$.

In the second article we work with strongly stationary ends with cubic volume growth and prove that they are WAF.

${ }^{2}$ We thank H Friedrich for pointing out this reference. 
Before ending this part of the introduction we would like to adventure a physical sentence. It appears from our findings that, granting GR to be correct and granting the stationarity of the exterior regions of galaxies, then the existence of dark matter seems to be an inevitable fact. In other words, it is not possible to explain the observed gravitational distortions around disc galaxies in terms of vacuum GR alone.

In the first part of the next section we explain the mathematical setup in which the main theorems are going to be proved. For this reason it can be thought as part of the background material. After the setup is introduced we return to give the precise statements to be proved (including the main result), and elaborate on open questions.

\subsection{The setup, the statements to prove and open questions}

In the following discussion we summarize the mathematics of strong stationary ends (M, $\mathbf{g}$ ) as seen in the quotient space. We refer to [12] for a detailed account on stationary solutions.

Let $E$ be the manifold that results from the quotient of $\mathbf{M}$ by $X$, let $\pi: \mathbf{M} \rightarrow E$ be the projection and let $\tilde{g}$ be the quotient three-metric. By (S1) $E$ is diffeomorphic to $\mathbb{R}^{3}$ minus an open ball and by $(\mathrm{S} 2)(E, \tilde{g})$ is geodesically complete until the boundary (recall that geodesics in $(E, \tilde{g})$ can be lifted to geodesics in $(\mathbf{M}, \mathbf{g})$ perpendicular to $X$ and preserving the arclength). All the relevant components of the Einstein equations can be written in the quotient space in terms of $\tilde{g}$, the 'norm' $u$ of the Killing $X$, i.e. $u:=\sqrt{-\langle X, X\rangle_{\mathbf{g}}}$, and the twist one-form $\omega$ which is defined by

$$
\omega=\pi_{*}\left(\star\left(\frac{1}{2} \xi \wedge \mathrm{d} \xi\right)\right),
$$

where $\star$ is the $\mathbf{g}$-Hodge star and the one-form $\xi$ is the $\mathbf{g}$-dual of the Killing, i.e. $\xi:=\langle X,\rangle_{\mathbf{g}}$. In $\mathbf{M}$ the metric $\mathbf{g}$ is $\mathbf{g}=-(\xi \otimes \xi) / u^{2}+\pi^{*} g$ and in terms of the data $(g, \omega, u)$ an isometric copy $(\hat{\mathbf{M}}, \hat{\mathbf{g}})$ of $(\mathbf{M}, \mathbf{g})$ can be obtained by making $\hat{\mathbf{M}}=\mathbb{R} \times E$ and $\hat{\mathbf{g}}=-u^{2}(\mathrm{~d} t+\theta)^{2}+\tilde{g}$, where here $t$ is the coordinate in the $\mathbb{R}$-factor and the one form $\theta$ (in $E$ ) is found by solving $\mathrm{d} \theta=-\star 2 \omega / u^{4}$ where $\star$ is the $\tilde{g}$-Hodge star.

In principle one can work with the variables $(\tilde{g}, \omega, u)$ but, as it turns out, the Einstein equations display a rich structure when expressed instead in terms of the conformally transformed metric

$$
g:=u^{2} \tilde{g}
$$

the form $\omega$ and the function $u$. In terms of $(g, \omega, u)$ the vacuum Einstein equations are equivalent to ([12])

$$
\left\{\begin{array}{l}
\text { Ric }=2 \nabla \ln u \otimes \nabla \ln u+\frac{2}{u^{4}} \omega \otimes \omega, \\
\Delta \ln u=-\frac{2|\omega|^{2}}{u^{4}}, \\
\operatorname{div} \omega=4\langle\nabla \ln u, \omega\rangle, \\
\mathrm{d} \omega=0,
\end{array}\right.
$$

where Ric is the Ricci tensor of $g, \Delta$ is the $g$-Laplacian, I I denotes $g$-norm and $\langle$,$\rangle denotes$ the $g$-inner product. In the last two equations div $\omega$ is the $g$-divergence of $\omega$ and $\mathrm{d} \omega$ its exterior derivative. The equations (1) enjoy remarkable structures which will be introduced as 
the article progresses. The data $(E ; \tilde{g}, \omega, u)$ or the equivalent data $(E ; g, \omega, u)$ will be called a stationary end when $(\mathbf{M}, \mathbf{g})$ is a stationary end and a strong stationary end when $(\mathbf{M}, \mathbf{g})$ is strongly stationary.

By $(\mathrm{S} 3)$ the space $(E, g)$ of a strongly stationary end is also geodesically complete (until the boundary). The analysis and the results of this article remain unchanged if instead of (S3) we impose just the completeness of $(E, g)$. We will recall this later when we comment corollary 1.9 .

To be explicit, the definition of asymptotic flatness with Schwarzschildian fall off that we adopt is the following (cf [4]).

Definition 1.4. Let $(E ; \tilde{g}, \omega, u)$ be a stationary end. Then, it is asymptotically flat with Schwazschildian fall off if there is a coordinate system $\left\{x=\left(x^{1}, x^{2}, x^{3}\right)\right\}$ covering $E$ up to a compact set such that

$$
\left|\delta_{i j}-\tilde{g}_{i j}\right| \leqslant K /|x|, \quad\left|\partial_{k} \tilde{g}_{i j}\right| \leqslant K /|x|^{2}, \quad\left|\partial_{m} \partial_{k} \tilde{g}_{i j}\right| \leqslant K /|x|^{3},
$$

and,

$$
\left|\partial_{i} u\right|+\left|\omega_{i}\right| \leqslant K /|x|^{2}
$$

plus further progressive power-law decay for the norms of the multiple $\partial$-derivatives of $\tilde{g}_{i j}, u$ and $\omega_{i}$, where here $K$ is a positive constant and $|x|$ is the norm of $x=\left(x^{1}, x^{2}, x^{3}\right)$ as a vector in $\mathbb{R}^{3}$.

The definition is the same if instead of $(E ; \tilde{g}, \omega, u)$ we consider $(E ; g, \omega, u)$.

We move now to state the main theorem (theorem 1.5) to be proved between part I (this article) and part II (the next article). The main result, which was stated in the previous section as theorem 1.3, will be deduced as a corollary of our main theorem. It is stated below again (for expository reasons), as corollary 1.6.

We recall first the definition of cubic volume growth. Denote by $\mathcal{T}_{g}(\partial E, r)$ the metric (tubular) neighborhood of $\partial E$ and radius $r>0$, that is, the set of points in $E$ at a distance (see section 2) from $\partial E$ less than $r$. Then, $(E, g)$ is said to have cubic growth if $\lim \operatorname{Vol}_{g}\left(\mathcal{T}_{g}(\partial E, r)\right) / r^{3}=\mu>0$. Note that the limit always exists as a result of the Bishop-Gromov monotonicity of $\operatorname{Vol}_{g}\left(\mathcal{T}_{g}(\partial E, r)\right) / r^{3}$ due to the non-negativity of the Ricci curvature of $g$ (first equation in (1)).

Between this article and its sequel we then give a proof of:

Theorem 1.5 (main theorem of parts I and II). Any strongly stationary end $(E ; g, \omega, u)$ having cubic volume growth is asymptotically flat with Schwarzschildian fall off.

Although we will keep including explicitly inside the statements that the strongly stationary solutions have cubic volume growth, it is a very important fact that this condition can be entirely removed from the statement of theorem 1.5 due to very general geometric facts. In this point we found it important to distinguish between what requires the full structure of stationary solutions and what is indeed a property of a much more general character, which, as a matter of fact, requires for its proof quite different techniques. The reason why this volumegrowth condition is unnecessary is the following. First and as remarked before, strongly stationary ends have non-negative Ricci curvature and also, as proved by M T Anderson [1], 
they have quadratic curvature decay. Second, it was proved in [9] that complete metrics in $\mathbb{R}^{3}$ minus a ball with non-negative Ricci curvature and quadratic curvature decay have cubic volume growth. Thus, the combination of these two facts shows that assuming cubic volume growth in theorem 1.5 is unnecessary. This conclusion is essentially the statement of our main result given in theorem 1.3. For the sake of a clearer exposition we restate this below as a corollary to the theorem 1.5 , this time in terms of the physical variables $(\tilde{g}, \omega, u)$.

Corollary 1.6 (main result, restatement). Any strongly stationary end $(E ; \tilde{g}, \omega, u)$ is asymptotically flat and has Schwarzschildian fall off.

The proof of theorem 1.5 is, as was advanced before, broken in two parts. In this first article we work with WAF ends and prove

Theorem 1.7 (main theorem of part I). Every WAF end is asymptotically flat with Schwarzschildian fall off.

In the second article instead we work with strongly stationary ends and prove

Theorem 1.8 (main theorem of part II). Every strongly stationary end having cubic volume growth is WAF.

A relevant open question left from this work is whether stationary spacetime ends are always strongly stationary or not. Or, in the light of corollary 1.6, it is open whether stationary spacetime ends are always AF or not. The following 'gap corollary' partially answers this question. To size the relevance of the result keep in mind the a priori estimates from [1] according to which there is a universal constant $\mathcal{K}>0$ (so far unknown) such that for any strongly stationary end $(E ; \tilde{g}, \omega, u)$ we have

$|(\nabla \ln u)(p)|_{\tilde{g}} \leqslant \frac{\mathcal{K}}{\operatorname{dist}_{\tilde{g}}(p, \partial E)}, \quad|\omega(p)|_{\tilde{g}} \leqslant \frac{\mathcal{K}}{\operatorname{dist}_{\tilde{g}}(p, \partial E)}, \quad$ and $\left|\operatorname{Ric}_{\tilde{g}}(p)\right|_{\tilde{g}} \leqslant \frac{\mathcal{K}}{\operatorname{dist}_{\tilde{g}}^{2}(p, \partial E)}$,

where $\operatorname{dist}_{\tilde{g}}(p, \partial E)$ is the $\tilde{g}$-distance from $p$ to $\partial E$ (see section 2 ).

Corollary 1.9. Let $(E ; \tilde{g}, \omega, u)$ be a stationary end. If

$$
|(\nabla \ln u)(p)|_{\tilde{g}} \leqslant \frac{1}{\operatorname{dist}_{\tilde{g}}(p, \partial E)}
$$

then the end is asymptotically flat with Schwazschildian fall off.

This shows that if one can prove that the universal constant $\mathcal{K}$ must be a priori less or equal than one then every stationary end is AF with Schwarzschildian fall off. The question is undoubtedly of fundamental importance. The proof of the corollary, whose details are left to the reader, is done by showing that under (2) we have $u(p) \geqslant c / \operatorname{dist}_{\tilde{g}}(p, \partial E)$ for some constant $c>0$ and for all $p$ with $\operatorname{dist}_{\tilde{g}}(p, \partial E) \geqslant 1$, and then observing from this that if $\tilde{g}$ is complete then so is $g=u^{2} \tilde{g}$. As commented before, this is enough to get the same conclusions as in corollary 1.5 .

The contents for the rest of this article are roughly the following. The definition of WAF ends is given in section 3. In section 3.1 we work out the main properties of WAF ends and reach the conclusion (proposition 3.5) that to prove that they are AF it is indeed enough to 
prove that the so called $\varepsilon$-flat ends (definition 3.6), which enjoy much nicer properties, are AF. This and the Schwazschildian fall off are proved in section 4 (proposition 4.6). This proves theorem 1.7 .

\section{Background material I}

We collect here the material required for the technical discussions. We introduce too the most relevant terminology and notation. From now on our main variables will be $(g, \omega, u)$.

\subsection{Distance}

- The distance between two points $p$ and $q$ in a connected manifold $(M, g)$ is $\operatorname{dist}_{g}(p, q)=\inf \left\{\right.$ length $_{g}\left(C_{p, q}\right): \quad C_{p, q}$ a $C^{1}$ curve in $M$ joining $p$ to $\left.q\right\} .(M, g)$ is said to be complete if $\left(M\right.$, dist $\left._{g}\right)$ is complete as a metric space. The distance from a point $p$ to a set $\Omega \subset M$ will be denoted by $\operatorname{dist}_{g}(p, \Omega)=\left\{\operatorname{dist}_{g}(p, q): q \in \Omega\right\}$. More generally, the distance between two sets $\Omega_{1}$ and $\Omega_{2}$ is denoted by $\operatorname{dist}_{g}\left(\Omega_{1}, \Omega_{2}\right)=\inf \left\{\operatorname{dist}_{g}(p, q): p \in \Omega_{1}, q \in \Omega_{2}\right\}^{3}$.

- The metric induced on stationary ends $(E, g)$ will be noted by $\operatorname{dist}(p, q)$ and always without the subindex $g$. The distance function to the boundary $\partial E$ of stationary ends will be denoted with total exclusivity by $d(p)$ or simply $d$, that is, $d(p)=\operatorname{dist}(p, \partial E)=\inf \{\operatorname{dist}(p, q): q \in \partial E\}$.

\subsection{Scaling}

- For any $r>0$ we will denote by $g_{r}$ to the scaled metric

$$
g_{r}:=\frac{1}{r^{2}} g
$$

Tensors and metric quantities constructed out of $g_{r}$ will be subindexed with an $r$. For instance, for the scalar curvature we have $R_{g_{r}}=R_{r}=R / r^{2}$ and for the Ricci curvature $R i c_{g_{r}}=R i c_{r}=R i c$ (although $R i c_{r}=R i c$ we will keep including the subindex $r$ ). Also, $d_{r}(p)=d(p) / r$. This way of notating will be used extensively throughout the article and is crucial in keeping track of it.

\subsection{Area, second fundamental form and mean curvature}

- The Riemannian metric induced on compact embedded surfaces $S \subset E$ will be denoted by $h$ and the $h$-area of $S$ by $A(S)$. Following the notation introduced before, the metric induced in $S$ from $g_{r}$ is denoted by $h_{r}$ and the $h_{r}$-area of $S$, i.e. $A(S) / r^{2}$, is denoted by $A_{r}(S)$.

- The second fundamental form of $S$ (fixed some normal) will be denoted by $\Theta$ and the mean curvature $\operatorname{tr}_{h} \Theta$ by $\theta$. Again, for the second fundamental form and the mean curvature of $S$ found from the scaled metric $g_{r}$ we will use $\Theta_{r}(=r \Theta)$ and $\theta_{r}(=\theta / r)$ respectively.

${ }^{3}$ Properly speaking this is not a metric in the subsets of $M$. In particular the distance is zero if for instance they share a point but are different sets. 


\subsection{Annuli and metric annuli}

- For any $0<a<b$ we will denote by $\mathcal{A}(a, b)$ (resp. $\mathcal{A}[a, b])$ the set $\mathcal{A}(a, b)=\{p \in E: a<d(p)<b\}, \quad(\operatorname{resp} . \mathcal{A}[a, b]=\{p \in E: a \leqslant d(p) \leqslant b\})$, and call it the open (resp. closed) metric annulus of radii a and $b$. The notation $\mathcal{A}(a, b)$ (resp. $\mathcal{A}[a, b]$ ) will always refer to open (resp. closed) metric annuli defined with respect to the unscaled metric $g$ but the subindex $r$ is included when the (open or closed) metric annuli are defined with respect to the scaled metric $g_{r}=g / r^{2}$, namely

$\mathcal{A}_{r}(a, b)=\left\{p \in E: a<d_{r}(p)<b\right\}, \quad$ and $\quad \mathcal{A}_{r}[a, b]=\left\{p \in E: a \leqslant d_{r}(p) \leqslant b\right\}$.

This is consistent with the notation introduced before. Note that for all $r>0$ we have $\mathcal{A}(a r, b r)=\mathcal{A}_{r}(a, b)$ and $\mathcal{A}[a r, b r]=\mathcal{A}_{r}[a, b]$.

- Standard open annuli in $\mathbb{R}^{3}$ will be denoted by $\mathcal{A}_{\mathbb{R}^{3}}(a, b)$, namely,

$$
\mathcal{A}_{\mathbb{R}^{3}}(a, b)=\left\{x \in \mathbb{R}^{3}: a<|x|<b\right\}=B_{\mathbb{R}^{3}}(o, b) \backslash \overline{B_{\mathbb{R}^{3}}(o, a)},
$$

where for any $c>0, B_{\mathbb{R}^{3}}(o, c)$ is the open ball of the center of the origin $o=(0,0,0)$ and radius $c$ in $\mathbb{R}^{3}$. As before closed annulus in $\mathbb{R}^{3}$ are denoted by $\mathcal{A}_{\mathbb{R}^{3}}[a, b]=\left\{x \in \mathbb{R}^{3}: a \leqslant|x| \leqslant b\right\}$.

- A manifold $\Omega$ is an open (resp. closed) annulus if $\Omega$ is diffeomorphic to $\mathcal{A}_{\mathbb{R}^{3}}(1,2)$ (resp. $\left.\mathcal{A}_{\mathbb{R}^{3}}[1,2]\right)$. A metric annulus doesn't have to be necessarily an open annulus in this sense. In general, the shape of the metric annuli can be wild.

All these notations will be used extensively.

\subsection{The Ernst equation}

- The second and third equations of (1) can be grouped in the so called Ernst equation. Let $\phi$ be a potential for $\omega$, i.e. $d \phi=\omega$. Define the complex function $\mathcal{E}=u^{2}+2 \phi i$. Then the Ernst equation is

$$
\Delta \mathcal{E}=2 \frac{\langle\nabla \mathcal{E}, \nabla \mathcal{E}\rangle}{\mathcal{E}+\mathcal{E}^{*}},
$$

where $\mathcal{E}^{*}$ is the complex conjugate. Making $\chi:=\nabla \mathcal{E}$ and $\zeta:=(\nabla \mathcal{E}) / u^{2}$, the equation (3) can be written in the form of the linear system in $\chi$,

$$
\left\{\begin{array}{l}
\operatorname{div} \chi=\langle\zeta, \chi\rangle, \\
d \chi=0,
\end{array}\right.
$$

where we are thinking $\zeta$ simply as a coefficient. This viewpoint of the Ernst equation will be important and will be recalled in the proof of (4.3).

\subsection{Curvature}

- A first fundamental property derived from the first equation in (1) is

$$
|R i c|^{2} \leqslant R^{2} \leqslant 2|R i c|^{2}
$$

which says that the scalar curvature $R$ fully controls the Ricci curvature Ric. Here $|R i c|^{2}=R i c_{a b} R i c^{a b}$. A second fundamental property is 


$$
\Delta R \geqslant R^{2}
$$

which says that the scalar curvature is subharmonic. The proof of (5) is given in [1] (pp 987-988) by manipulating the Bochner-type of the formula for the energy density $e=R$ of the harmonic map $\mathcal{E}=\left(2 \phi, u^{2}\right)$ from $E$ into the half space model of the hyperbolic two-space $\mathrm{H}^{2}=\left(\{(x, y): y>0\},\left(\mathrm{d} x^{2}+\mathrm{d} y^{2}\right) / y^{2}\right)$.

- Another essential property of the curvature of stationary solutions is M T Anderson's a priori curvature decay [1]. Applied to ends it says that there is a universal constant $\mathcal{K}>0$ such that for any strong stationary end $E$ we have ${ }^{4}$,

$$
|\operatorname{Ric}(p)| \leqslant \mathcal{K} / d^{2}(p) .
$$

In particular for any $p \in \mathcal{A}_{r}(a, b)$, the Ricci curvature of the scaled metric $g_{r}$ is bounded as $\left|\operatorname{Ric}_{r}(p)\right|_{r} \leqslant \mathcal{K} / a^{2}$.

\subsection{Norms and convergence of Riemannian manifolds}

- Given a tensor field $U$ (of any valence) on a region $\Omega$ of a manifold $(M, g)$, the $C_{g}^{i}$-norm of $U$ over $\Omega$ is defined as

$$
\|U\|_{C_{g}^{i}(\Omega)}:=\sup _{p \in \Omega} \sum_{j=0}^{j=i}\left|\left(\nabla^{j} U\right)(p)\right|_{g} .
$$

Of course $\|U\|_{C_{g}^{i}} \leqslant\|U\|_{C_{g}^{i+1}}$. The subindex $g$ will be suppressed when $\Omega$ is a region of the Euclidean three-space, namely we will write $C^{i}$.

- All that we will need about convergence of smooth Riemannian manifolds will be contained in the following definition (which is not the most general [8]). Let $\left(\Omega_{m}, g_{m}\right)$ be a sequence of smooth, compact, connected three-manifolds with smooth boundary and let $\left(\Omega_{\infty}, g_{\infty}\right)$ also be smooth, compact, connected three-manifold with smooth boundary. Then, $\left(\Omega_{m}, g_{m}\right)$ converges to $\left(\Omega_{\infty}, g_{\infty}\right)$ in $C^{i}, i \geqslant 2$, if there are diffeomorphisms $\varphi_{m}: \Omega_{\infty} \rightarrow \Omega_{m}$ such that $\left\|\varphi_{m}^{*} g_{m}-g_{\infty}\right\|_{{C_{\infty}}^{i}\left(\Omega_{\infty}\right)} \rightarrow 0$ where $\varphi_{m}^{*} g_{m}$ is the pull-back of $g_{m}$ by $\varphi_{m}$. The definition is the same if we do not require compactness on the $\Omega_{m}$ and $\Omega_{\infty}$ but assume uniformly bounded diameters. A sequence of smooth tensors $U_{m}$ converge to a smooth tensor $U_{\infty}$ in $C^{i}, i \geqslant 0$,if $\left\|\varphi_{m}^{*} U_{m}-U_{\infty}\right\|_{C_{g_{\infty}}^{i}\left(\Omega_{\infty}\right)} \rightarrow 0$.

\subsection{Elliptic estimates}

- Stationary solutions $(M ; g, \omega, u)$ satisfy important regularity properties derived directly from the elliptic system (1).

Proposition 2.1. Let $(M ; g, \omega, u)$ be a stationary solution and let $p \in M$. Suppose that all over $M$ we have $R \leqslant R_{0}$ and that at $p$ we have inj $(p) \geqslant I_{0}>0$ and $u(p)+1 / u(p) \leqslant \Lambda_{0}$, where $\operatorname{inj}_{g}(p)$ is the injectivity radius at $p$. Then, for any $i \geqslant 0$ there are constants $K_{1}^{i}\left(R_{0}, I_{0}\right)>0$ and $K_{2}^{i}\left(R_{0}, I_{0}, \Lambda_{0}\right)>0$ such that

\footnotetext{
4 There is a caveat here. The curvature estimate provided in theorem 0.2 of [1] is (as written) for the spacetime metric and not for the metric $g$. However the proof of that theorem is achieved by proving first the estimate $\left|\operatorname{Ric}_{g}(p)\right| \leqslant \mathcal{K} / \operatorname{dist}_{g}^{2}(p, \partial M)$ (see cf step I in [1]), that is all that we need here.
} 


$$
\| \text { Ric } \|_{C_{g}^{i}(B)} \leqslant K_{1}^{i}, \quad \text { and } \quad\|\omega\|_{C_{g}^{i}(B)}+\|u\|_{C_{g}^{i+1}(B)} \leqslant K_{2}^{i},
$$

where $B=B_{g}\left(p, I_{0} / 2\right)$.

It is important to remark that the constants $K_{1}^{i}$ which bound the $C_{g}^{i}$-norm of Ric do not depend on $\Lambda_{0}$. This is because any stationary solution can be scaled to the stationary solution $(g, \bar{\omega}, \bar{u})=\left(g, \omega / u(p)^{2}, u / u(p)\right)$ which has $\bar{u}(p)=1$.

In part I, not much will be required about elliptic estimates because most of the necessary estimates are already contained in the definition of the WAF end. The following however is a simple application that will be used in the proof of proposition 4.3. Let $\left(\Omega_{m} ; g_{m}, \omega_{m}, u_{m}\right)$ be a sequence of stationary solutions and suppose that $\left(\Omega_{m}, g_{m}\right)$ converges in $C^{2}$ to the flat annulus $\left(\mathcal{A}_{\mathbb{R}^{3}}(a, b), g_{\mathbb{R}^{3}}\right)$. Then, $g_{m}$ converges to $g$ also in $C^{i}$ for any $i \geqslant 2$ and without the necessity of taking a subsequence. Moreover there are scalings $\bar{\omega}_{m}:=\lambda_{m}^{2} \omega_{m}$ and $\bar{u}_{m}:=\lambda_{m} u_{m}$ such that $\bar{\omega}_{m}$ and $\bar{u}_{m}$ converge in $C^{i-2}$ and $C^{i-1}$ to the one-form zero and the constant function one, respectively. In particular the scale invariant one-forms $\zeta_{m}=2\left(u_{m} \nabla u_{m}+i \omega_{m}\right) / u_{m}^{2}$ converge in $C^{i-1}$ to the one-form zero.

\section{WAF ends}

Definition 3.1. A stationary end $E$ is WAF if it is strongly stationary and for every $i \geqslant 2$, $l \geqslant 1$ and divergent sequence $r_{m} \rightarrow \infty$, there is a sequence of open annuli $\Omega_{m} \subset E$ such that:

(W1) $\mathcal{A}_{r_{m}}\left(1 / 2,2^{l}\right) \subset \Omega_{m}$ for every $m$.

(W2) $\left(\Omega_{m}, g_{r_{m}}\right)$ converges in $C^{i}$ to the flat annulus $\left(\mathcal{A}_{\mathbb{R}^{3}}\left(1 / 2,2^{l}\right), g_{\mathbb{R}^{3}}\right)$.

(W3) The scaled distance functions $d_{r_{m}}$ (restricted to $\Omega_{m}$ ) converge in $C^{0}$ to the distance to the origin in $\mathbb{R}^{3}$ (restricted to $\mathcal{A}_{\mathbb{R}^{3}}\left(1 / 2,2^{l}\right)$ ).

(W4) Every $\bar{\Omega}_{m}$ is a closed annulus and separates $\partial E$ from infinity, namely, $\partial E$ belongs to a bounded component of $E \backslash \bar{\Omega}_{m}$ for all $m$.

Figure 3 illustrates a WAF end together with some annuli $\Omega_{m}$.

It can be shown that the condition (W4) is in fact redundant. Namely, if we define WAF ends exactly as in definition 3.1 but removing (W4), then any such end would also comply with definition 3.1 including (W4). The proof of that is not relevant to us and is skipped.

To be clear from the start, let us recall that the convergences in (W2) and (W3) express the existence of diffeomorphisms $\varphi_{m}: \mathcal{A}_{\mathbb{R}^{3}}\left(1 / 2,2^{l}\right) \rightarrow \Omega_{m}$ such that the metrics $\varphi_{m}^{*} g_{r_{m}}$ over $\mathcal{A}_{\mathbb{R}^{3}}\left(1 / 2,2^{l}\right)$ converge in $C^{i}$ to the flat Euclidean metric and that, at the same time, the functions $d_{r_{m}}{ }^{\circ} \varphi_{m}$ over $\mathcal{A}_{\mathbb{R}^{3}}\left(1 / 2,2^{l}\right)$ converge in $C^{0}$ to the distance function to the origin.

We stress that in (W2) the convergence is for the entire sequence $\left(\Omega_{m}, g_{r_{m}}\right)$ and not just for a subsequence of it. Note also that by (W3) the sets $\varphi_{m}^{-1}\left(\mathcal{A}_{r_{m}}\left(1 / 2,2^{l}\right)\right)$ tend to cover the whole $\mathcal{A}_{\mathbb{R}^{3}}\left(1 / 2,2^{l}\right)$, in the sense that for every $1 / 2>\varepsilon>0$ there is $m_{\varepsilon}$ such that if $m \geqslant m_{\varepsilon}$ then $\mathcal{A}_{\mathbb{R}^{3}}\left(1 / 2+\varepsilon, 2^{l}-\varepsilon\right) \subset \varphi_{m}^{-1}\left(\mathcal{A}_{r_{m}}\left(1 / 2,2^{l}\right)\right)$ The reason why we need the regions $\Omega_{m}$ in 


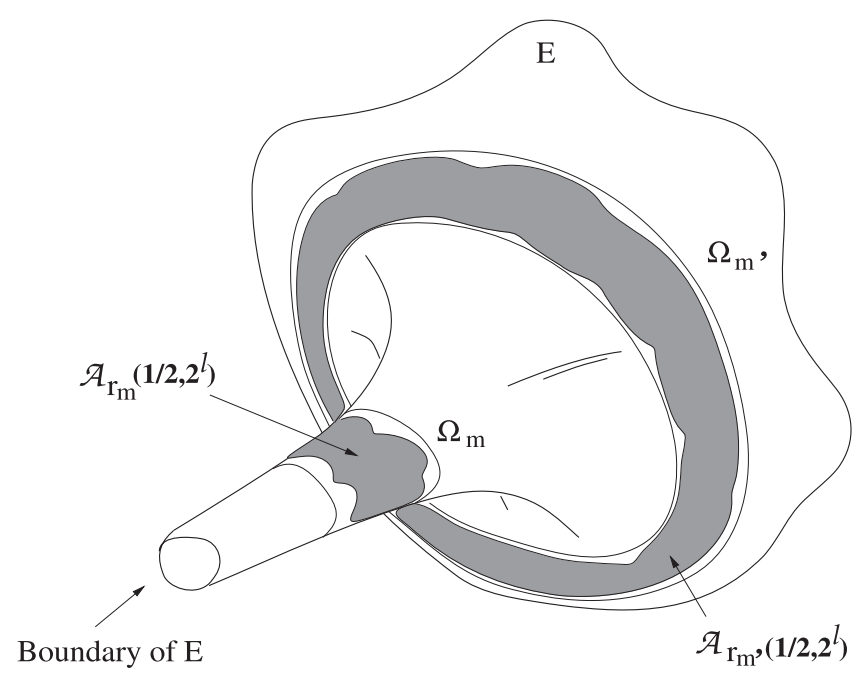

Figure 3. Representation of a WAF end along with the annuli $\Omega_{m}$ and the metric annuli $\mathcal{A}_{r_{m}}\left(1 / 2,2^{l}\right)$.

definition 3.1 is essentially technical and not particularly important. As we show in part II, strong stationary ends with cubic volume growth are WAF in the sense of definition 3.1 and, to stick to the definition of convergence, the existence of the diffeomorphisms $\varphi_{m}$ is guaranteed only into the regions $\Omega_{m}$ (which cover tightly the metric annuli $\mathcal{A}_{r_{m}}\left(1 / 2,2^{l}\right)$ ) but unfortunately not into the metric annuli $\mathcal{A}_{r_{m}}\left(1 / 2,2^{l}\right)$.

WAF ends have a number of technical advantages allowing us to prove the standard Schwarzschildian decay in a more comfortable setup. The next section discusses some of the basic properties of WAF ends.

\subsection{Preliminaries of WAF ends}

The final goal of this section is to prove proposition 3.5. This proposition says that one can always restrict the domain of a given WAF end $E$ by removing from it an annulus (with one boundary component $\partial E$ ) to get an end simpler to handle for its nice geometric properties. This type of WAF ends, which are defined in definition 3.6, we call $\varepsilon$-flat.

The first proposition says that the curvature of WAF ends decays faster than quadratically.

Proposition 3.2. Let $E$ be a WAF end. Then, for any divergent sequence of points $p_{m}$ (i.e. $\left.d\left(p_{m}\right) \rightarrow \infty\right)$ we have

$$
\lim _{m \rightarrow \infty}\left|\operatorname{Ric}\left(p_{m}\right)\right| d^{2}\left(p_{m}\right)=0 .
$$

Thus, if we write $|\operatorname{Ric}(p)|=o(p) / d^{2}(p)$, then $o(p) \rightarrow 0$ when $d(p) \rightarrow \infty$.

Proof. Let $p_{m}$ be any divergent sequence of points and let $r_{m}=d\left(p_{m}\right)$. From the definition of the WAF end (with $l=1$ and $i=2$ ), there is a sequence of regions $\Omega_{m}$, with 
$p_{m} \in \mathcal{A}_{r_{m}}(1 / 2,2) \subset \Omega_{m}$ for every $m$, such that $\left(\Omega_{m}, g_{r_{m}}\right)$ converges in $C^{2}$ to a flat annulus. Therefore $\left|\operatorname{Ric}_{r_{m}}\left(p_{m}\right)\right|_{r_{m}} \rightarrow 0$, or, equivalently, $\left|\operatorname{Ric}\left(p_{m}\right)\right| d^{2}\left(p_{m}\right) \rightarrow 0$.

Proposition 3.3. Let $E$ be a WAF end. Then, for every $\delta>0$ there is $r_{\delta}>0$ and a twosphere $S_{\delta}$ separating $\partial E$ from infinity such that:

(Al) $1-\delta \leqslant \inf \left\{d_{r_{\delta}}(p): p \in S_{\delta}\right\} \leqslant \sup \left\{d_{r_{\delta}}(p): p \in S_{\delta}\right\} \leqslant 1+\delta$.

(A2) For all $p \in S_{\delta}$ we have $\left|\hat{\Theta}_{r_{\delta}}(p)\right|_{r_{\delta}} \leqslant \delta$ and $\left|\theta_{r_{\delta}}(p)-2\right| \leqslant \delta$.

(A3) For all $p$ in the unbounded component $E_{S_{\delta}}$ of $E \backslash S_{\delta}$ we have

$$
\left|\operatorname{Ric}_{r_{\delta}}(p)\right|_{r_{\delta}} \leqslant \frac{\delta}{\left(1+\operatorname{dist}_{r_{\delta}}\left(p, S_{\delta}\right)\right)^{2}} .
$$

We recall from the notation in section 2 that in (A2) above, $\hat{\Theta}_{r_{\delta}}$ is the traceless part of the second fundamental form $\Theta_{r_{\delta}}$ and $\theta_{r_{\delta}}$ is the mean curvature of the surface $S_{\delta}$ as a surface embedded in the Riemannian space $\left(E, g_{r_{\delta}}\right)$ (hence the subindex $r_{\delta}$ ). To define $\Theta_{r_{\delta}}$ we assume a normal in $S_{\delta}$ inwards to $E_{S_{\delta}}$.

Proof. Assume at the moment that $0<\delta<1 / 4$. This simplifies the algebra a little. We remove it at the end. We start observing that, as the curvature of the WAF stationary ends decays faster than quadratically, then:

(i) For every $0<\delta<1 / 4$ there is $\hat{r}_{\delta}>0$ such that for every $p$ with $d(p) \geqslant \hat{r}_{\delta}$ we have $|\operatorname{Ric}(p)| \leqslant \delta / 4 d^{2}(p)$ (the 4 in the denominator is also for algebraic convenience).

Second, we observe that using definition 3.1 (with $i=2$ and $l=1$ ) we can guarantee that:

(ii) For every $0<\delta<1 / 4$ there is $r_{\delta} \geqslant 2 \hat{r}_{\delta}\left(\hat{r}_{\delta}\right.$ as in $\left.(i)\right)$, an open annulus $\Omega \supset \mathcal{A}_{r_{\delta}}(1 / 2,2)$, and a diffeomorphism $\varphi: \mathcal{A}_{\mathbb{R}^{3}}(1 / 2,2) \rightarrow \Omega$ such that the metric $\varphi^{*} g_{r_{\delta}}$ is sufficiently close in $C^{2}$ to the euclidean metric and the function $d_{r_{\delta}} \circ \varphi$ is sufficiently close in $C^{0}$ to the distance function to the origin in $\mathbb{R}^{3}$, that if we define $S_{\delta}:=\varphi\left(\partial B_{\mathbb{R}^{3}}(o, 1)\right)$ then (Al) and (A2) hold.

Thus, with this definition of $S_{\delta}$ we have already (A1) and (A2). That $S_{\delta}$ separates $\partial E$ from infinity is direct because, by (W4), $\bar{\Omega}$ is a closed annulus in $E$ separating $\partial E$ from infinity ${ }^{5}$. It remains to prove (A3). We do that now. For any $p \in E_{S_{\delta}}$ (the unbounded component of $E \backslash E_{S_{\delta}}$ ) we have

$$
d(p) \geqslant \operatorname{dist}\left(p, S_{\delta}\right)+\operatorname{dist}\left(S_{\delta}, \partial E\right) .
$$

To see this consider a geodesic segment from $p$ to $\partial E$ whose length realizes $d(p)$. Such a segment must intersect $S_{\delta}$ in (at least) one point $q$. Hence, $d(p)=\operatorname{dist}(p, q)+\operatorname{dist}(q, \partial E) \geqslant \operatorname{dist}\left(p, S_{\delta}\right)+\operatorname{dist}\left(S_{\delta}, \partial E\right)$ as wished. Dividing the expression (6) by $r_{\delta}$ we obtain $d_{r_{\delta}}(p) \geqslant \operatorname{dist}_{r_{\delta}}\left(p, S_{\delta}\right)+\operatorname{dist}_{r_{\delta}}\left(S_{\delta}, \partial E\right)$ and from this and (A1) we get

5 It is an exercise in topology to prove that, as $E \sim \mathbb{R}^{3} \backslash B_{\mathbb{R}^{3}}(o, 1)$ and as $\bar{\Omega}$ is a closed annulus embedded in $E$ separating $\partial E$ from infinity, then $E \backslash \Omega$ consists of two pieces, one of which is a closed annulus containing $\partial E$ and the other which is diffeomorphic to $\mathbb{R}^{3} \backslash B_{\mathbb{R}^{3}}(o, 1)$. 


$$
d_{r_{\delta}}(p) \geqslant \operatorname{dist}_{r_{\delta}}\left(p, S_{\delta}\right)+1-\delta .
$$

Observe then that as $0<\delta<1 / 4$ we have $d_{r_{\delta}}(p) \geqslant 1-\delta>1 / 2$ and thus $d(p)>r_{\delta} / 2$. But as in (ii) we assumed $r_{\delta} \geqslant 2 \hat{r}_{\delta}$ then $d(p) \geqslant \hat{r}_{\delta}$ and we can use (i). Now use (7) in $\left|\operatorname{Ric}_{r_{\delta}}(p)\right|_{r_{\delta}} \leqslant \delta / 4 d_{\delta}^{2}(p)$, which we obtain from (i) by scaling $|\operatorname{Ric}(p)| \leqslant \delta / 4 d^{2}(p)$, to deduce

$$
\left|\operatorname{Ric}_{r_{\delta}}(p)\right|_{r_{\delta}} \leqslant \frac{\delta / 4}{\left(d_{r_{\delta}}(p)+1-\delta\right)^{2}} \leqslant \frac{\delta}{\left(\operatorname{dist}_{r_{\delta}}\left(p, S_{\delta}\right)+1\right)^{2}},
$$

where the second inequality holds as long as $0<\delta<1 / 4$ as we are assuming. This shows (A3) as wished.

When $\delta \geqslant 1 / 4$ then define $r_{\delta}:=r_{1 / 8}$ and $S_{\delta}:=S_{1 / 8}$. With this definition (A1)-(A3) are immediate because $\delta>1 / 8$ (for instance $\left|\hat{\Theta}_{r_{\delta}}(p)\right|_{r_{\delta}}=\left|\hat{\Theta}_{r_{1 / 8}}(p)\right|_{r_{1 / 8}} \leqslant 1 / 8 \leqslant \delta$ ).

Let $(M, g)$ be a complete Riemannian manifold with $M$ diffeomorphic to $\mathcal{R}^{3}$ minus a ball. At least for a small $t_{*}>0$, the equidistant sets $\mathcal{S}(t):=\{p \in M$, $\operatorname{dist}(p, \partial M)=t\}$, where $t \leqslant t_{*}$, are embedded spheres. For any $p \in \mathcal{S}(t)$ with $0 \leqslant t \leqslant t_{*}$ denote by $\theta(p)$ and by $\hat{\Theta}(p)$ the mean curvature and traceless part of the second fundamental form of $\mathcal{S}(t)$ at $p$ (in the direction of increasing $t$ ) respectively. We will use this notation (cf 'second fundamental form' in section 2) in the statement of the next proposition which will be used later in conjunction with proposition 3.3 to deduce proposition 3.5. In the item (B2) inside the statement below we let $\mathrm{Q}>0$ be any numeric constant such that $|R m| \leqslant 4 \mathrm{Q} \mid$ Ric $\mid$ (recall that in dimension three the Ricci curvature determines the Riemann curvature by an algebraic formula). The constant $\varrho$ plays some algebraic role later but is not particularly important.

Proposition 3.4. For any $\varepsilon>0$ there is $\delta>0$ such that if a complete smooth Riemannian three-manifold $(M, g)$, with $M$ diffeomorphic to $\mathbb{R}^{3}$ minus a ball, satisfies

(B1) $|\theta(p)-2| \leqslant \delta$ and $|\hat{\Theta}(p)| \leqslant \delta$ for all $p \in \partial M$, and,

$(B 2)|R i c(p)| \leqslant \delta /\left(\varrho(1+\operatorname{dist}(p, \partial M))^{2}\right)$ for all $p \in M$, then,

$$
\left|\theta(p)-\frac{2}{1+t}\right| \leqslant \frac{\varepsilon}{1+t}, \quad \text { and } \quad|\widehat{\Theta}(p)| \leqslant \frac{\varepsilon}{1+t},
$$

as long as $p \in S(t)=\{p$ : $\operatorname{dist}(p, \partial M)=t\}$ and $t \leqslant t_{*}$, where $t_{*}>0$ is such that for any $t \in\left[0, t_{*}\right]$ the equidistant sets $S(t)$ are embedded surfaces.

That $M$ is diffeomorphic to $\mathbb{R}^{3}$ minus a ball will play no role in the proof. Despite this we will keep this assumption for several expository reasons.

Proof. Let $\mathcal{F}$ be the congruence of geodesics in $M$ emanating perpendicularly from $\partial M$. We will denote such geodesics as $\gamma_{q}(t), q \in \partial M$. Observe that if $\gamma_{q}(t) \in S\left(t^{\prime}\right)$ then $t=t^{\prime}$. For this reason we would be done if we prove that over every geodesic $\gamma_{q}(t)$ we have $\left|\theta\left(\gamma_{q}(t)\right)-2 /(1+t)\right| \leqslant \varepsilon /(1+t)$ and $\left|\hat{\Theta}\left(\gamma_{q}(t)\right)\right| \leqslant \varepsilon /(1+t)$. This is exactly how we will proceed and to do so we will study a couple of differential inequalities that $\theta$ and $|\hat{\Theta}|$ satisfy along the geodesics $\gamma_{q}(t)$ (equations (12) and (13)) to obtain upper and lower 
bounds for $\theta\left(\gamma_{q}(t)\right)$ and $\left|\hat{\Theta}\left(\gamma_{q}(t)\right)\right|$ essentially equivalent to (8). We move first to deduce these differential inequalities ((12) and (13)).

In the computations below $h(t)$ denotes the Riemannian-metric induced over $S(t)$. Recall that the Lie derivative of $\Theta$ along the velocity field ${ }^{6} n$ of the congruence $\mathcal{F}$ is given by

$$
\Theta^{\prime}=\Theta \circ \Theta-\operatorname{Rm}(n, n),
$$

where $(\Theta \circ \Theta)_{a b}:=\Theta_{a}^{c} \Theta_{c b}$ and where $R m(n, n)$ is the symmetric two-form $\operatorname{Rm}(n, n)(v, w):=\operatorname{Rm}(v, n, w, n)$. The contraction of this equation gives, as is well known, the focusing equation

$$
\theta^{\prime}=-\frac{\theta^{2}}{2}-|\widehat{\Theta}|^{2}-\operatorname{Ric}(n, n)
$$

This equation and the ones below are to be evaluated along the geodesics $\gamma_{q}$ but we will omit writing this dependence explicitly for notational convenience. Also, for later advantage we will use $\bar{t}:=1+t$ instead of $t$ as the parameter of the geodesics $\gamma_{q}$. We claim now that from (9) and (10) we obtain the equation

$$
|\widehat{\Theta}|^{2 \prime}=-2\langle\widehat{\Theta}, \widehat{\Theta} \circ \widehat{\Theta}\rangle-2 \theta|\widehat{\Theta}|^{2}+2\langle\widehat{\Theta}, \widehat{R m}(n, n)\rangle,
$$

where $\hat{R m}(n, n)$ is the traceless part of $R m(n, n)$ and where $\langle, \quad\rangle$ is the inner product among symmetric two-tensors defined by $\langle U, V\rangle=U_{a b} V_{c d} h^{a c} h^{b c}$. To see this we compute

$$
\begin{aligned}
\widehat{\Theta}^{\prime} & =\Theta^{\prime}-\frac{\theta^{\prime}}{2} h-\theta \Theta \\
& =\Theta \circ \Theta-\operatorname{Rm}(n, n)+\left(\frac{\theta^{2}}{2}+|\widehat{\Theta}|^{2}+\operatorname{Ric}(n, n)\right) \frac{h}{2}-\theta \Theta \\
& =\widehat{\Theta} \circ \widehat{\Theta}+\frac{|\widehat{\Theta}|^{2}}{2} h-\widehat{R m}(n, n),
\end{aligned}
$$

where to obtain the first equality we use that $h^{\prime}=2 \Theta$. Therefore

$$
\begin{aligned}
|\widehat{\Theta}|^{2 \prime} & =2\left\langle\widehat{\Theta}, \widehat{\Theta}^{\prime}\right\rangle-4\langle\Theta, \widehat{\Theta} \circ \widehat{\Theta}\rangle \\
& =2\left\langle\widehat{\Theta}, \widehat{\Theta} \circ \widehat{\Theta}+\frac{|\widehat{\Theta}|^{2}}{2} h-\widehat{R m}(n, n)\right\rangle-4\langle\Theta, \widehat{\Theta} \circ \widehat{\Theta}\rangle \\
& =-2\langle\widehat{\Theta}, \widehat{\Theta} \circ \widehat{\Theta}\rangle-2\langle\widehat{R m}(n, n), \widehat{\Theta}\rangle-2 \theta|\widehat{\Theta}|^{2},
\end{aligned}
$$

as wished ${ }^{7}$. The equations (11) and (10) give the inequalities

$$
\begin{aligned}
& |\widehat{\Theta}|^{2 \prime} \leqslant 2|\widehat{\Theta}|^{3}-2 \theta|\widehat{\Theta}|^{2}+2|\widehat{\Theta}||\widehat{R m},(n, n)|, \\
& -\frac{\theta^{2}}{2}-|\widehat{\Theta}|^{2}-|\operatorname{Ric}(n, n)| \leqslant \theta^{\prime} \leqslant-\frac{\theta^{2}}{2},
\end{aligned}
$$

which hold along every geodesic $\gamma_{q}(\bar{t})$. We will analyze them in what follows under the hypothesis (B1) and (B2). We will then adjust $\delta$ to satisfy (8) for the given $\varepsilon$. As a matter of

${ }^{6}$ That is, $n\left(\gamma_{q}(t)\right)=\gamma_{q}^{\prime}(t)$.

7 To deduce the first equality in this calculation use $\langle\Theta, \Theta\rangle=\Theta_{a b} \Theta_{c d} h^{a c} h^{b d}$ and $\left(h^{a b}\right)^{\prime}=-\left(h_{c d}{ }^{\prime}\right) h^{c a} h^{b d}=-2 \Theta^{a b}$. 
fact the first adjustment of $\delta$ is $\delta \leqslant \min \{\varepsilon, 1 / 32\}$ that will be assumed from now on. The reason for this will become clear later.

One can easily get a first conclusion just analyzing the second inequality in (13). Indeed from it, $\delta \leqslant \epsilon$ and (B1), we deduce that $\theta(\bar{t})$ satisfies

$$
\left\{\begin{array}{l}
\theta^{\prime} \leqslant-\frac{\theta^{2}}{2} \\
\theta(1) \leqslant 2+\varepsilon .
\end{array}\right.
$$

One can then directly compare $\theta(\bar{t})$ to $x(\bar{t})=(2+\varepsilon) / \bar{t}$ because $x(\bar{t})$ satisfies

$$
\left\{\begin{array}{l}
x^{\prime} \geqslant-\frac{x^{2}}{2} \\
x(1)=2+\varepsilon
\end{array}\right.
$$

to conclude, from a standard ODE analysis, that $x(\bar{t})$ is an upper barrier to $\theta(\bar{t})$, that is $\theta(\bar{t}) \leqslant(2+\varepsilon) / \bar{t}$. Hence to show (8) it remains to be proven that one can adjust $\delta$ further to also have

$$
\frac{2-\varepsilon}{\bar{t}} \leqslant \theta(\bar{t}), \quad \text { and } \quad|\widehat{\Theta}(\bar{t})| \leqslant \frac{\varepsilon}{\bar{t}} .
$$

The proof of these two bounds is simultaneous and is done at the end of the discussion below.

- Assume that for any $\bar{t} \in\left[1, \bar{t}_{a}\right]$ we have $\theta(\bar{t}) \geqslant 3 / 2 \bar{t}$. In the analysis below we will be restricted to this interval of $\bar{t}$. Now use this assumption and (B2) in (12) to get

$$
|\widehat{\Theta}|^{2 \prime} \leqslant 2|\widehat{\Theta}|^{3}-\frac{3}{\bar{t}}|\widehat{\Theta}|^{2}+\frac{2 \delta}{\bar{t}^{2}}|\widehat{\Theta}|,
$$

where we deduce the last term on the rhs use in addition that

$|\widehat{R m}(n, n)|=\left|\operatorname{Rm}(n, n)-\left(\operatorname{tr}_{h} \operatorname{Rm}(n, n)\right) \frac{h}{3}\right| \leqslant 4|\operatorname{Rm}(n, n)| \leqslant 4 \varrho|\operatorname{Ric}(n, n)| \leqslant \delta / \bar{t}^{2}$,

by (B2) and by how the constant $Q$ was defined (see the definition before the statement of the proposition). Consider then the first order ODE

$$
\left(x^{2}\right)^{\prime}=2 x^{3}-\frac{3}{\bar{t}} x^{2}+\frac{2 \hat{\delta}}{\bar{t}^{2}} x
$$

which is obtained by making $|\hat{\Theta}|=x=x(\bar{t})$ in (15), then changing the inequality by an equality and finally changing $\delta$ by $\hat{\delta}$ which we assume to satisfy $\delta \leqslant \hat{\delta} \leqslant \min \{\varepsilon, 1 / 32\}^{8}$. The reason why we consider this ODE is the following: if $x(\bar{t})$ is a positive solution to (16) such that $x(1) \geqslant|\hat{\Theta}(1)|$ then $x(\bar{t}) \geqslant|\hat{\Theta}(\bar{t})|$ as long as they are defined. We now look for a solution to (16) of the form $x=a / \bar{t}$, where $a$ is a constant. Substituting $x=a / \bar{t}$ in (16) we obtain

$$
-2 a^{2} \frac{1}{\bar{t}^{3}}=\left(2 a^{3}-3 a^{2}+2 \hat{\delta} a\right) \frac{1}{\bar{t}^{3}} .
$$

Canceling the factor $a / \bar{t}^{3}$ and solving for $a$ we obtain that $x=a / \bar{t}(a \neq 0)$ is a solution to (16) iff $a$ satisfies

${ }^{8}$ To obtain an ODE of the form $x^{\prime}=F(x, \bar{t})$ use $\left(x^{2}\right)^{\prime}=2 x x^{\prime}$ and then divide by $2 x$ in (16). 


$$
a=\frac{1}{4}(1 \pm \sqrt{1-16 \hat{\delta}})
$$

For us only the solution corresponding to the smaller $a$ will be important, namely $a=a_{-}:=(1-\sqrt{1-16 \hat{\delta}}) / 4$. Note for later reference that $a_{-}>0$ and that $a_{-} \rightarrow 0$ as $\hat{\delta} \rightarrow 0$.

With the solution $x=a_{-} / \bar{t}$ at hand we can obtain the following first conclusion.

- If $|\hat{\Theta}(1)| \leqslant a_{-}$and $\theta(1) \geqslant 3 / 2$, then $|\hat{\Theta}(\bar{t})| \leqslant a_{-} / \bar{t}$ as long as $\theta(\bar{t}) \geqslant 3 / 2 \bar{t}$.

$\circ$ Assume now that for any $\bar{t} \in\left[1, \bar{t}_{b}\right]$ we have $|\hat{\Theta}(\bar{t})| \leqslant a_{-} / \bar{t}$ (where $a_{-}(\hat{\delta})$ is as before).

In the analysis below we will be restricted to this interval of $\bar{t}$. Use this assumption and (B2) in the inequality (13) to get

$$
\theta^{\prime} \geqslant-\frac{\theta^{2}}{2}-\frac{\left(a_{-}^{2}+\delta\right)}{\bar{t}^{2}} .
$$

Consider then the first order ODE

$$
x^{\prime}=-\frac{x^{2}}{2}-\frac{\left(a_{-}^{2}+\hat{\delta}\right)}{\bar{t}^{2}},
$$

which is obtained by making $\theta=x$ in (18), then changing the inequality by an equality and finally changing $\delta$ by $\hat{\delta}$. Again, the reason why we consider this ODE is the following: if $x(\bar{t})$ is a solution to (19) such that $x(1) \leqslant \theta(1)$ then $x(\bar{t}) \leqslant \theta(\bar{t})$ as long as they are defined. We look now for a solution to (19) of the form $x=b / \bar{t}$ where $b$ is a constant. Substituting $x=b / \bar{t}$ in (19) we obtain

$$
-b \frac{1}{\bar{t}^{2}}=\left(-\frac{b^{2}}{2}-\left(a_{-}^{2}+\hat{\delta}\right)\right) \frac{1}{\bar{t}^{2}} .
$$

Canceling the factor $1 / \bar{t}^{2}$ and solving for $b$ we deduce that $b / \bar{t}$ is a solution to (19) iff $b$ satisfies

$$
b=1 \pm \sqrt{1-2\left(a_{-}^{2}+\hat{\delta}\right)}
$$

The solution $b_{+}$will be the only important one. Note for reference below that $b_{+}<2$ and $b_{+} \rightarrow 2$ as $\hat{\delta} \rightarrow 0$.

With the solution $x=b / \bar{t}$ at hand we can obtain the following second conclusion.

- If $\theta(1) \geqslant b_{+}$and $|\hat{\Theta}(1)| \leqslant a_{-}$, then $\theta(\bar{t}) \geqslant b_{+} / \bar{t}$ as long as $|\hat{\Theta}(\bar{t})| \leqslant a_{-} / \bar{t}$.

We proceed to combine both conclusions to finally adjust $\delta$ to satisfy (14). Choose $\hat{\delta}$ smaller than $\min \{\varepsilon, 1 / 32\}$ if necessary to have

$$
a_{-}(\hat{\delta}) \leqslant \varepsilon, \quad \text { and } \quad b_{+}(\hat{\delta})>\max \left\{2-\varepsilon, \frac{3}{2}\right\} .
$$

Then we make the choice

$$
\delta=\min \left\{\hat{\delta}, a_{-}(\hat{\delta}), 2-b_{+}(\hat{\delta})\right\}
$$


Observe that with this choice of $\delta$, the hypothesis (B1) implies

$$
\theta(1) \geqslant 2-\delta \geqslant b_{+}>2-\varepsilon, \quad \text { and } \quad|\widehat{\Theta}(1)| \leqslant \delta \leqslant a_{-}(\hat{\delta}) \leqslant \varepsilon .
$$

We claim that for all $\bar{t} \in\left[1,1+t_{*}\right]$ we have

$$
\theta(\bar{t}) \geqslant \frac{b_{+}}{\bar{t}}, \quad \text { and } \quad|\widehat{\Theta}(\bar{t})| \leqslant \frac{a_{-}}{\bar{t}},
$$

that would imply (14) because $b_{+}>2-\varepsilon$ and $a_{-} \leqslant \varepsilon$. Hence we would be done after proving the claim. We do that below.

Suppose the claim is false and let $\bar{t}_{F}<t_{*}+1$ be the last time for which both inequalities in (21) hold.

Case 1. Suppose there are times $\bar{t}$ greater than $\bar{t}_{F}$ but arbitrarily close to it for which $|\hat{\Theta}(\bar{t})|>a_{-} / \bar{t}$. Let $\bar{t}_{F}^{\prime}>\bar{t}_{F}$ close enough to $\bar{t}_{F}$ that on $\left[\bar{t}_{F}, \bar{t}_{F}^{\prime}\right]$ we still have $\theta(\bar{t})>3 / 2 \bar{t}$. According to the first conclusion there must be a time $\bar{t}_{1}$ before $\bar{t}_{F}{ }^{\prime}$ for which $\theta\left(\bar{t}_{1}\right)<3 / 2 \bar{t}_{1}$ which is not possible.

Case 2. Suppose instead that there is $\bar{t}^{\prime}{ }_{F}$ such that on $\left[\bar{t}_{F}, \bar{t}^{\prime}{ }_{F}\right]$ we still have $|\hat{\Theta}(\bar{t})| \leqslant a_{-} / \bar{t}$ but that there are times $\bar{t}$ greater than $\bar{t}_{F}$ but arbitrarily close to it for which $\theta(\bar{t})<b_{+} / \bar{t}$. Then according to the second conclusion there must be a time $\bar{t}_{1}$ less than $\bar{t}_{F}^{\prime}$ for which $\left|\hat{\Theta}\left(\bar{t}_{1}\right)\right|>a_{-} / \bar{t}$, which is not possible.

Proposition 3.5. Let $E$ be a WAF end. Then for every $0<\varepsilon<1 / 2$ there is $r_{\varepsilon}>0$ and an embedded two sphere $S_{\varepsilon}$ separating $\partial E$ from infinity, such that

(U1) On the unbounded component $E_{S_{\varepsilon}}$ of $E \backslash S_{\varepsilon}$ the distance function $\operatorname{dist}_{r_{\varepsilon}}\left(p, S_{\varepsilon}\right)$ is smooth and every level set $S(t)=\left\{p\right.$ : $\left.\operatorname{dist}_{r_{\varepsilon}}\left(p, S_{\varepsilon}\right)=t\right\}$ is an embedded sphere, and,

(U2) For every $p \in S(t)$ we have

$\left|\widehat{\Theta}_{r_{\varepsilon}}(p)\right|_{r_{\varepsilon}} \leqslant \frac{\varepsilon}{1+t}, \quad\left|\theta_{r_{\varepsilon}}(p)-\frac{2}{1+t}\right| \leqslant \frac{\varepsilon}{1+t}, \quad$ and $\quad\left|\operatorname{Ric}_{r_{\varepsilon}}(p)\right|_{r_{\varepsilon}} \leqslant \frac{\varepsilon}{(1+t)^{2}}$.

The condition $\varepsilon<1 / 2$ is not relevant for the proof but helps for algebraic reasons. It is also included for compatibility with definition 3.6, which is motivated by proposition 3.5 and where the condition is required.

Proof. For the given $\varepsilon$ denote by $\delta^{\varepsilon}$ the delta provided by proposition 3.4. Then, by proposition 3.3 one can find for every $\delta \leqslant \min \left\{\delta^{\varepsilon} / \mathrm{\varrho}, \varepsilon\right\}$ a sphere $S_{\delta}$ and a $r_{\delta}$ such that (A1)-(A3) hold. Here $\varrho$ is the numeric constant defined before the statement of proposition 3.4. Hence (B1) and (B2) hold too if we define the manifold $(M, g)$ as $\left(E_{S_{\delta}}, g_{r_{\delta}}\right)$ and we can use proposition 3.4. If we define $r_{\varepsilon}$ (the one claimed by the proposition) as $r_{\varepsilon}:=r_{\delta}$, we conclude using (8) that (U2) will be valid as long as $t \in\left[0, t_{\delta}^{*}\right.$ ) where $t_{\delta}^{*}$ is the supremum of the $t_{*}>0$ such that for every $t \in\left[0, t_{*}\right]$ the set $S(t)=\left\{p \in E_{S_{\delta}}: \operatorname{dist}_{r_{\delta}}\left(p, S_{\delta}\right)=t\right\}$ is an embedded sphere.

Observe that this construction is valid for every $\delta \leqslant\left\{\delta^{\varepsilon}, \varepsilon\right\}$ and also that given $\delta$ we can choose $r_{\delta}$ (and therefore $r_{\varepsilon}$ ) larger than any given number (by a simple inspection of the proof 
of proposition 3.3). To prove (U1) it is enough to show that we can choose $\delta$ small enough and then $r_{\delta}$ big enough to have $t_{\delta}^{*}=\infty$. To this purpose it suffices to show that for any sequences $\delta^{i} \downarrow 0$ (with $\delta^{i=0} \leqslant \min \left\{\delta^{\varepsilon}, \varepsilon\right\}$ ) and $r_{\delta^{i}} \uparrow \infty$ there is some $i$ for which $t_{\delta^{i}}^{*}=\infty$. Consider then one such pair of sequences and suppose, arguing by contradiction, that $t_{\delta^{i}}^{*}$ is finite for every $i$. We will show that this is impossible. From the construction of the spheres $S_{\delta^{i}}$ (cf (ii) inside the proof of proposition 3.3) there is, for every $i$, an annulus $\Omega_{i} \supset \mathcal{A}_{r_{\delta^{i}}}(1 / 2,2)$ together with a diffeomorphism $\varphi_{i}: \mathcal{A}_{\mathbb{R}^{3}}(1 / 2,2) \rightarrow \Omega_{i}$, both provided by the definition of WAF end, and with $\varphi_{i}\left(\partial B_{\mathbb{R}^{3}}(o, 1)\right)=S_{\delta^{i}}$. As $r_{\delta^{i}} \rightarrow \infty$, then (also from the definition of WAF end) the metrics $\varphi_{i}^{*} g_{r_{\delta^{i}}}$ converge in $C^{2}$ to the Euclidean metric and the functions $d_{r_{\delta^{i}}} \circ \varphi_{i}$ converge in $C^{0}$ to the distance function to the origin. But in $\mathbb{R}^{3}$ the equidistant sets $\left\{x \in \mathcal{A}_{\mathbb{R}^{3}}(1,2)\right.$ : $\left.\operatorname{dist}_{\mathbb{R}^{3}}\left(x, \partial B_{\mathbb{R}^{3}}(o, 1)\right)=t\right\}$ are obviously equal to the embedded spheres $\partial B_{\mathbb{R}^{3}}(o, 1+t)$ for all $t \in[0,1)$. Therefore, by continuity, there is $i_{0}$ such that if $i \geqslant i_{0}$ then $t_{\delta^{i}}^{*} \geqslant 1 / 2$. We then assume from now on and without loss of generality that $t_{\delta^{i}}^{*} \geqslant 1 / 2$ for every $i \geqslant 0$.

By (U2) and (for every $i$ ) the mean curvature of the spheres $S(t)$ remains finite for every $t \in\left[0, t_{\delta^{i}}^{*}\right]$. Thus (for every $i$ ) the surfaces $S(t)$ are embedded when $t<t_{\delta^{i}}^{*}$ but just at $t=t_{\delta^{i}}^{*}$ the surface $S\left(t_{\delta^{i}}^{*}\right)$ is only immersed. We conclude that (for every $i$ ) there is at least a point $p_{i}^{*}$ in $S\left(t_{\delta^{i}}^{*}\right)$ of self tangency of $S\left(t_{\delta^{i}}^{*}\right)$ (see figure 4). As the surfaces $S(t)$ are equidistant to $S_{\delta^{i}}$ it is deduced that at $p_{i}^{*}$ and at $t=t_{\delta^{i}}^{*}$ there arrive two geodesic segments $\gamma_{1}^{i}(t)$ and $\gamma_{2}^{i}(t)$ (i.e. $\left.\gamma_{1}^{i}\left(t_{\delta^{i}}^{*}\right)=\gamma_{2}^{i}\left(t_{\delta^{i}}^{*}\right)\right)$ that start at $S_{\delta^{i}}$ when $t=0$. Moreover the geodesics $\gamma_{1}^{i}(t)$ and $\gamma_{2}^{i}(t) \operatorname{cross}$ $S\left(t^{\prime}\right)$ only at $t=t^{\prime}$ and do so perpendicularly. For these reasons when they reach $p_{i}^{*}$ they do so with opposite velocities, that is $\gamma_{1}{ }^{\prime}\left(t_{\delta^{i}}^{*}\right)=-\gamma_{2}{ }^{\prime}\left(t_{\delta^{i}}^{*}\right)$, and moreover we have $\operatorname{dist}_{r_{\delta}}\left(\gamma_{1}(t), S_{\delta}\right)=\operatorname{dist}_{r_{\delta}}\left(\gamma_{2}(t), S_{\delta}\right)=t$ for all $t \in\left[0, t_{\delta^{i}}^{*}\right]$. These geodesic segments are depicted in figure 4. Note that one can form a larger geodesic segment, denoted here by $\gamma^{i}$, simply by concatenating $\gamma_{1}^{i}$ and $\gamma_{2}^{i}$ at $p_{i}^{*}$. The distance $r_{i}^{*}:=d\left(p_{i}^{*}\right)$ will be important below. Also it will be useful to parametrize $\gamma^{i}$ with the $g_{r_{i}^{*}}$ (signed) arc length $\tau^{i}$ starting from $p_{i}^{*}$ (in one of the two directions). As $t$ (which is a $g_{r_{\delta^{i}}}$-arc length) ranges in $\left[0, t_{\delta^{i}}^{*}\right]$ then $\tau^{i}$ ranges in $\left[-\left(r_{i}^{*} / r_{\delta^{i}}\right) t_{\delta^{i}}^{*},\left(r_{i}^{*} / r_{\delta^{i}}\right) t_{\delta^{i}}^{*}\right]$. We show later that $r_{i}^{*} / r_{\delta^{i}} \geqslant 1$. From this and $t_{\delta^{i}}^{*} \geqslant 1 / 2$ we deduce that $\tau^{i}$ ranges at least in $[-1 / 2,1 / 2]$.

To reach a contradiction we will use the following inequality

$$
\left|d_{r_{i}^{*}}\left(\gamma^{i}\left(\tau^{i}\right)\right)-d_{r_{i}^{*}}\left(\gamma^{i}\left(-\tau^{i}\right)\right)\right| \leqslant 2 \delta^{i} \frac{r_{\delta^{i}}}{r_{i}^{*}} .
$$

We also prove this later but for the moment and to avoid much disruption we proceed to use it. By the definition of the WAF end we can consider a sequence of annuli $\Omega_{i} \supset \mathcal{A}_{r_{i}^{*}}(1 / 2,2)$ together with the sequence of diffeomorphisms $\varphi_{i}: \mathcal{A}_{\mathbb{R}^{3}}(1 / 2,2) \rightarrow \Omega_{i}$ such that $\varphi_{i}^{*} g_{r_{i}^{*}}$ converges in $C^{2}$ to the Euclidean metric and such that $d_{r_{i}^{*} \circ} \circ \varphi_{i}$ converges in $C^{0}$ to the distance function to the origin. Taking a subsequence if necessary, the pull back of the geodesics 


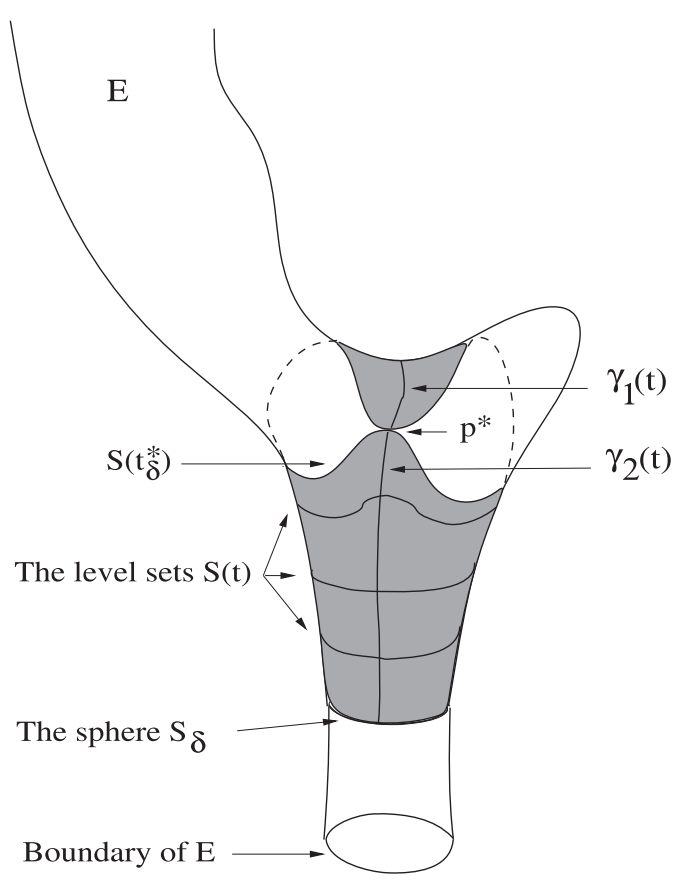

Figure 4. Representation of the geometric elements in the proof by contradiction of (U1) in proposition 3.5. For simplicity we do not include the index ' $i$ '.

segments $\gamma^{i}\left(\tau^{i}\right)$ converge to a geodesic segment $\gamma(\tau)$ in $\mathbb{R}^{3}$ (hence a straight segment) with $|\gamma(\tau=0)|=1$ because $1=d_{r_{i}^{*}}\left(p_{i}^{*}=\gamma^{i}(0)\right) \rightarrow|\gamma(0)|($ here $|\gamma(\tau)|$ is the norm of $\gamma(\tau)$ as a point in $\mathbb{R}^{3}$, hence the Euclidean distance from $\gamma(\tau)$ to the origin). Moreover

$$
|| \gamma(\tau)|-| \gamma(-\tau)||=0, \quad \text { for all } \tau \in\left[-\frac{1}{2}, \frac{1}{2}\right],
$$

by taking the limit of (22) (observe when taking the limit that $r_{\delta^{i}} / r_{i}^{*} \leqslant 1$ ). This equality is clearly impossible if $|\gamma(0)|=1$ and we reach a contradiction.

To finish the proof it remains to prove (22) and also $r_{i}^{*} / r_{\delta^{i}} \geqslant 1$. We first show (22). To simplify the notation make below $\delta=\delta^{i}, r_{\delta}=r_{\delta^{i}}, r_{i}^{*}=r^{*}, t_{\delta^{i}}^{*}=t_{\delta}^{*}, \gamma_{1}=\gamma_{1}^{i}$ and $\gamma_{2}=\gamma_{2}^{i}$. In this (22) is equivalent to

$$
\left|d_{r^{*}}\left(\gamma_{1}(t)\right)-d_{r^{*}}\left(\gamma_{2}(t)\right)\right| \leqslant 2 \delta \frac{r_{\delta}}{r^{*}} .
$$

We claim that this follows from proving, for any $p \in E_{S_{\delta}}$, the inequality

$$
\operatorname{dist}_{r_{\delta}}\left(p, S_{\delta}\right)+1-\delta \leqslant d_{r_{\delta}}(p) \leqslant \operatorname{dist}_{r_{\delta}}\left(p, S_{\delta}\right)+1+\delta .
$$

Indeed, if in it we make $p=\gamma_{j}(t), j=1,2$ and then use that $\operatorname{dist}_{r_{\delta}}\left(\gamma_{j}(t), S_{\delta}\right)=t$ for $j=1,2$, we get

$$
t+1-\delta \leqslant d_{r_{\delta}}\left(\gamma_{j}(t)\right) \leqslant t+1+\delta, \quad \text { for } j=1,2
$$


and therefore

$$
(t+1-\delta) \frac{r_{\delta}}{r^{*}} \leqslant d_{r^{*}}\left(\gamma_{j}(t)\right) \leqslant(t+1+\delta) \frac{r_{\delta}}{r^{*}}, \quad \text { for } j=1,2
$$

from which (23) directly follows. We now deduce (24). Let $Y$ be a geodesic segment from $p$ to $\partial E$ whose length realizes the distance $d_{r_{\delta}}(p) . \Upsilon$ intersects $S_{\delta}$ in a point that we denote by $q$. Then we have

$$
d_{r_{\delta}}(p)=\operatorname{dist}_{r_{\delta}}(p, q)+d_{r_{\delta}}(q) \geqslant \operatorname{dist}_{r_{\delta}}\left(p, S_{\delta}\right)+1-\delta
$$

because $\operatorname{dist}_{r_{\delta}}(p, q) \geqslant \operatorname{dist}_{r_{\delta}}\left(p, S_{\delta}\right)$ and because $d_{r_{\delta}}(q) \geqslant 1-\delta$ by (A1). This shows the first inequality in (24). To show the second consider a point $q^{\prime} \in S_{\delta}$ such that $\operatorname{dist}_{r_{\delta}}\left(p, q^{\prime}\right)=\operatorname{dist}_{r_{\delta}}\left(p, S_{\delta}\right)$. Then

$$
d_{r_{\delta}}(p) \leqslant \operatorname{dist}_{r_{\delta}}\left(p, q^{\prime}\right)+d_{r_{\delta}}\left(q^{\prime}\right) \leqslant \operatorname{dist}_{r_{\delta}}\left(p, S_{\delta}\right)+1+\delta,
$$

because $d_{r_{\delta}}\left(q^{\prime}\right) \leqslant 1+\delta$ by (A1) again. This shows the second inequality in (24) as wished.

Finally we prove that $r^{*} / r_{\delta} \geqslant 1$. To see this use (24) with $p=p^{*}$ to get $t_{\delta}^{*}+1-\delta \leqslant r^{*} / r_{\delta}$ and the recall that $t_{\delta}^{*} \geqslant 1 / 2$ and $\delta \leqslant 1 / 2$.

The proposition 3.5 shows that one can always restrict the domain of a given end and then scale the metric out to obtain an end with better asymptotic properties. More concretely one can alway cut out $E$ at $S_{\delta}$ and then define an new end consisting of the resulting unbounded region $E_{S_{\delta}}$ and the scaled metric $g_{r_{\delta}}$. Of course if this new end is AF with Schwarzschildian decay so is the original end $E$. This shows that in order to prove asymptotic flatness for WAF ends it is enough to prove it for $\varepsilon$-flat ends defined as follows.

Definition 3.6. Let $\varepsilon$ be a number in $(0,1 / 2)$. Then, a stationary end $E$ is $\varepsilon$-flat if it is WAF and moreover:

(H1) The distance function $d(p)$ is smooth and every level set $S(r)=\{p: d(p)=r\}$ is an embedded sphere, and,

(H2) For every $p \in S(r)$ we have

$$
|\widehat{\Theta}(p)| \leqslant \frac{\varepsilon}{1+r}, \quad\left|\theta(p)-\frac{2}{1+r}\right| \leqslant \frac{\varepsilon}{1+r} \quad \text { and } \quad|\operatorname{Ric}(p)| \leqslant \frac{\varepsilon}{(1+r)^{2}} .
$$

Note that with respect to proposition 3.5 we have changed notation from $t$ to $r$. Also note that on $\varepsilon$-flat ends, metric annuli are indeed annuli (i.e. diffeomorphic to $\mathcal{A}_{\mathbb{R}^{3}}(1,2)$, cf section 2).

\section{Standard fall off for $\varepsilon$-flat ends}

As $\varepsilon$-flat stationary ends are just stationary ends with some additional properties we will continue using the same notations that we have used until now. 
Proposition 4.1. Let $E$ be an $\varepsilon$-flat end. Then, for all $p$ with $d(p) \geqslant 2 / \varepsilon-1$ we have

$$
\left|(\Delta d)(p)-\frac{2}{d(p)}\right| \leqslant \frac{2 \varepsilon}{d(p)} .
$$

Proof. Recall that $\Delta d=\theta$. Then, at a point $p$ with $d(p) \geqslant 2 \varepsilon-1$ we compute

$$
\begin{aligned}
\left|(\Delta d)(p)-\frac{2}{d(p)}\right| & \leqslant\left|(\Delta d)(p)-\frac{2}{d(p)+1}\right|+\left|\frac{2}{1+d(p)}-\frac{2}{d(p)}\right| \\
& \leqslant \frac{\varepsilon}{1+d(p)}+\frac{2}{(1+d(p)) d(p)} \leqslant\left(\frac{d(p)}{1+d(p)}+\frac{2}{\varepsilon(1+d(p))}\right) \frac{\varepsilon}{d(p)} \\
& \leqslant \frac{2 \varepsilon}{d(p)},
\end{aligned}
$$

where to obtain the second inequality we use $(\mathrm{H} 2)$ and to obtain the last we use $d(p) \geqslant 2 / \varepsilon-1$.

An important conclusion coming out of this proposition is that if we let $\alpha:=1-2 \varepsilon$ then the function $1 / d^{\alpha}$ is superharmonic on the region $\{p: d(p) \geqslant 2 / \varepsilon-1\}$, namely,

$$
\left(\Delta \frac{1}{d^{\alpha}}\right)(p) \leqslant 0
$$

for any $p$ such that $d(p) \geqslant 2 / \varepsilon-1$. To see this we compute

$$
\Delta \frac{1}{d^{\alpha}}=\frac{\alpha(\alpha+1)}{d^{\alpha+2}}-\frac{\alpha}{d^{\alpha+1}} \Delta d,
$$

and then use

$$
\Delta d \geqslant \frac{2-2 \varepsilon}{d}
$$

which is deduced from (25), to obtain

$$
\Delta \frac{1}{d^{\alpha}} \leqslant \frac{\alpha(2 \varepsilon+(\alpha-1))}{d^{2+\alpha}}=0
$$

as wished. We will use this below to deduce an important property of the scalar curvature $R$. For any $r \geqslant 0$ let

$$
\bar{R}(r):=\sup \{R(p): p \in S(r)\},
$$

be the supremum of $R$ over $S(r)$. As the scalar curvature $R$ decays quadratically at infinity then so does $\bar{R}(r)$. For this reason if $\bar{R}(r)$ is not monotonically decreasing, namely if there are $r_{1}<r_{2}$ such that $\bar{R}\left(r_{1}\right)<\bar{R}\left(r_{2}\right)$, then $R$ must have a local maximum somewhere. But as $\Delta R \geqslant R^{2}$ such local maximum cannot exist. We conclude that $\bar{R}(r)$ must be monotonically decreasing in $r$. In particular if $\bar{R}\left(r_{1}\right)=0$ for some $r_{1}$ then it is also zero for any $r \geqslant r_{1}$ in which case the stationary solution is simply a piece of the Minkowski spacetime. We will assume therefore from now on that $\bar{R}(r)>0$ for all $r \geqslant 0$. 
Proposition 4.2. Let $E$ be an $\varepsilon$-flat end and let $\alpha=1-2 \varepsilon$. Then, for any $r$ and $\bar{r}$ with $2 / \varepsilon-1 \leqslant \bar{r} \leqslant r$ we have

$$
\bar{R}(r) \leqslant\left(\frac{\bar{r}}{r}\right)^{\alpha} \bar{R}(\bar{r}) .
$$

Proof. As explained before the function $1 / d^{\alpha}$ is superharmonic on the region $\{p: d(p) \geqslant \bar{r}\}$ and therefore so is $1 / d_{\bar{r}}^{\alpha}$. Moreover the function $1 / d_{\bar{r}}^{\alpha}$ is identically one on $S(\bar{r})=\partial\{p: d(p)=\bar{r}\}$ and decreases to zero at infinity. On the other hand as $R$ is subharmonic so is $R / \bar{R}(\bar{r})$. Moreover the function $R / \bar{R}(\bar{r})$ is less or equal than one all over the set $S(\bar{r})$ and tends to zero at infinity. We can then compare the functions $1 / d_{\bar{r}}^{\alpha}$ and $R / \bar{R}(\bar{r})$ on the region $\{p: d(p) \geqslant \bar{r}\}$ using the maximum principle to conclude that $1 / d_{\bar{r}}^{\alpha}$ is greater than or equal to $R / \bar{R}(\bar{r})$ on $\{p: d(p) \geqslant \bar{r}\}$ everywhere. Hence

$$
R(p) \leqslant\left(\frac{\bar{r}}{r}\right)^{\alpha} \bar{R}(\bar{r}),
$$

from which (26) follows.

Proposition 4.3. Let $E$ be an $\varepsilon$-flat end. Then, there is a constant $c>0$ for which the following statement holds: for any $k \geqslant 1$ there is $r_{k}$ such that for any $\bar{r}$ and $\hat{r}$, with $r_{k} \leqslant \bar{r}$ and $\bar{r} \leqslant \hat{r} \leqslant 2^{k} \bar{r}$, we have

$$
\bar{R}(\hat{r}) \leqslant c\left(\frac{\bar{r}}{\hat{r}}\right)^{4} \bar{R}(\bar{r}) .
$$

This proposition is the basis to show that the scalar curvature $R$ has a $4-\eta$ decay for any $\eta>0$. Note that the constant $c$ is independent of $k$. We prove such decay in the following lemma. The proof of (4.3) is given after proving the lemma and the auxiliary proposition 4.5 .

Lemma 4.4. [(4- $\eta$-decay] Let $E$ be an $\varepsilon$-flat end. Then, given $\eta>0$ there exists $c_{\eta}>0$ such that

$$
\bar{R}(r) \leqslant \frac{c_{\eta}}{r^{4-\eta}}
$$

for any $r>0$. In particular at any point $p$ we have

$$
R(p) \leqslant \frac{c_{\eta}}{d(p)^{4-\eta}}
$$

Proof of lemma 4.4. Let $k \geqslant 1$, let $r_{k}$ be as in proposition 4.3 and let $r$ be any number greater than or equal to $2^{k} r_{k}$. Write $r$ in the form

$$
r=2^{k l} r_{*},
$$


where $l \geqslant 1$ is an integer and $r_{*} \in\left[r_{k}, 2^{k} r_{k}\right]$. For every $m$ from 1 to $l$ obtain

$$
\bar{R}\left(2^{m k} r_{*}\right) \leqslant \frac{c}{2^{4 k}} \bar{R}\left(2^{(m-1) k} r_{*}\right) \text {, }
$$

by using proposition 4.3 with $(\bar{r}, \hat{r})=\left(2^{(m-1) k} r_{*}, 2^{m k} r_{*}\right)$ and directly from them deduce

$$
\bar{R}(r)=\bar{R}\left(2^{k l} r_{*}\right) \leqslant\left(\frac{c}{2^{4 k}}\right)^{l} \bar{R}\left(r_{*}\right)=c^{l}\left(\frac{r_{*}}{r}\right)^{4} \bar{R}\left(r_{*}\right) .
$$

Note that $l=\left(\ln r / r_{*}\right) /(k \ln 2)$ and that therefore

$$
c^{l}=\left(\frac{r}{r_{*}}\right)^{(l \ln c) /\left(\ln r / r_{*}\right)}=\left(\frac{r}{r_{*}}\right)^{(\ln c) /(k \ln 2)} .
$$

Plugging (30) in (29) we obtain

$$
\bar{R}(r) \leqslant\left(\frac{r_{*}}{r}\right)^{4-(\ln c) /(k \ln 2)} \bar{R}\left(r_{*}\right) .
$$

Now choose $k$ large enough to have $\eta \geqslant(\ln c) /(k \ln 2)$. With this choice of $k$ and as $r_{*} / r \leqslant 1$ we obtain from (31) the inequality

$$
\bar{R}(r) \leqslant\left(\frac{r_{*}}{r}\right)^{4-\eta} \bar{R}\left(r_{*}\right)
$$

which is valid as long as $r \geqslant 2^{k} r_{k}$. Define now $c_{\eta}:=\max \left\{r_{*}^{4-\eta} \bar{R}\left(r_{*}\right): r_{*} \in\left[r_{k}, 2^{k} r_{k}\right]\right\}$. With this choice of $c_{\eta}$ the inequality (32) implies (28) for $r \geqslant 2^{k} r_{k}$. Finally increase $c_{\eta}$ if necessary to have (28) valid also for $r \in\left(0,2^{k} r_{k}\right)$.

The following proposition will be used only inside the proof of proposition 4.3 and is given separately for the sake of a smoother exposition.

Proposition 4.5. Let $\psi$ be a one form in $\mathbb{R}^{3} \backslash B_{\mathbb{R}^{3}}(o, 2)$, solution of

$$
\left\{\begin{array}{l}
\operatorname{div} \psi=0, \\
\mathrm{~d} \psi=0,
\end{array}\right.
$$

and satisfying that,

(al') $|\psi(x)| \leqslant 2 /|x|^{\alpha}$ for any $x$ in $\mathbb{R}^{3} \backslash B_{\mathbb{R}^{3}}(o, 2)$, and for some $\alpha>0$, and,

(a2') The $C^{1}$-norm of $\psi$ over $\mathcal{A}_{\mathbb{R}^{3}}[2,4]$ is bounded by $c^{*}$. Then, there is a constant $\hat{c}$ depending only on $c^{*}$ such that for all $x \in \mathbb{R}^{3} \backslash B_{\mathbb{R}^{3}}(o, 2)$ we have,

$$
|\psi(x)| \leqslant \frac{\hat{c}}{4|x|^{2}} .
$$

The factor 1/4 in (34) and radii 2 and 4 of the balls do not play any important role in the proposition but will be algebraically convenient when we use proposition 4.5 in proposition 4.3. 
Proof. Consider the real function $\hat{\varsigma}: \mathbb{R} \rightarrow \mathbb{R}$ defined by

$$
\hat{\varsigma}(y)= \begin{cases}0 & \text { if } y \in(-\infty, 2] \cup[4, \infty), \\ e^{-1 /(y-2)(4-y)} & \text { if } y \in(2,4),\end{cases}
$$

and then define the function $\varsigma: \mathbb{R} \rightarrow \mathbb{R}$ as

$$
\varsigma(y)=\frac{\int_{-\infty}^{y} \hat{\varsigma}(\bar{y}) \mathrm{d} \bar{y}}{\int_{-\infty}^{\infty} \hat{\varsigma}(\bar{y}) \mathrm{d} \bar{y}}
$$

The function $\varsigma(y)$ is just a non-negative $C^{\infty}$ function taking the value zero for $y \leqslant 2$ and the value one for $y \geqslant 4$. Then consider a potential function $\phi$ for $\psi$ on $\mathbb{R}^{3} \backslash B_{\mathbb{R}^{3}}(o, 2)$ which is simply found by integration along paths and that is unique up to a constant. Then, consider the function $\hat{\phi}(x):=\varsigma(|x|) \phi(x)$ as a function in the whole $\mathbb{R}^{3}$. This function satisfies

$$
\Delta \hat{\phi}=f
$$

where $f$ is a function with support in $B_{\mathbb{R}^{3}} \overline{(o, 4)}$ and whose $C^{1}$-norm is bounded by a constant $c_{1}^{*}\left(c^{*}\right)$. One can then represent $\hat{\phi}$ as the sum of a harmonic function $\hat{\phi}_{H}$ in $\mathbb{R}^{3}$ plus the solution $\hat{\phi}_{G}$ to (35) found by convoluting $f$ against the Green function of the Laplacian. The function $\hat{\phi}_{G}$ satisfies

$$
\left|\hat{\phi}_{G}(x)\right| \leqslant \frac{c_{2}^{*}}{1+|x|}, \quad \text { and } \quad\left|d \hat{\phi}_{G}(x)\right| \leqslant \frac{c_{3}^{*}}{(1+|x|)^{2}}
$$

where $c_{2}^{*}$ and $c_{3}^{*}$ depend only on $c^{*}$. Thus, as $\psi(x)=\mathrm{d} \hat{\phi}_{G}(x)+\mathrm{d} \hat{\phi}_{H}(x)$ when $|x| \geqslant 4$ and as by (a1') $|\psi(x)| \rightarrow 0$ when $|x| \rightarrow \infty$, we conclude that $\left|d \hat{\phi}_{H}(x)\right| \rightarrow 0$ when $|x| \rightarrow \infty$. In particular the harmonic functions $\partial_{x^{i}} \hat{\phi}_{H}, i=1,2,3$, also decay to zero at infinity. By Liouville's theorem the functions $\partial_{x} \hat{\phi}_{H}$ must be identically zero, from which we conclude that $\hat{\phi}_{H}$ is a constant and that $\psi(x)=\mathrm{d} \hat{\phi}_{G}(x)$ when $|x| \geqslant 4$. Define now $\hat{c}:=\max \left\{4 c_{3}^{*}, 64 c^{*}\right\}$. Then, by (36), if $|x| \geqslant 4$ we have $|\psi(x)| \leqslant c_{3}^{*} /|x|^{2} \leqslant \hat{c} / 4|x|^{2}$, while, by (a2'), if $2 \leqslant|x| \leqslant 4$ we have $|\psi(x)| \leqslant c^{*} \leqslant c / 4|x|^{2}$. Thus $|\psi(x)| \leqslant c / 4|x|^{2}$ for any $|x| \geqslant 2$ as wished.

Now we have all that is necessary to prove proposition 4.3 .

Proof of proposition 4.3. In what follows we assume $k \geqslant 1$ to be given and fixed. The proof of the proposition will rely on the use of the Ernst equation. The strategy of proof will be better explained once we state and prove facts (I), (II) and (III) below.

(I) For every given integers $i \geqslant 2$ and $j \geqslant 0$ and divergent sequence $\bar{r}_{m} \rightarrow \infty$ there is a sequence of annuli $\Omega_{m} \supset \mathcal{A}_{\bar{r}_{m}}\left(1 / 2,2^{k+j+2}\right)$ such that $\left(\Omega_{m}, g_{\bar{r}_{m}}\right)$ converges in $C^{i}$ to the flat annulus $\left(\mathcal{A}_{\mathbb{R}^{3}}\left(1 / 2,2^{j+k+2}\right), g_{\mathbb{R}^{3}}\right)$. In particular 


$$
\sup \left\{R_{\bar{r}_{m}}(p): p \in \mathcal{A}_{\bar{r}_{m}}\left(1,2^{k+j+2}\right)\right\} \rightarrow 0 .
$$

Moreover the complex one-forms $\zeta=2(u \nabla u+i \omega) / u^{2}$ (restricted to $\mathcal{A}_{\bar{r}_{m}}\left(1 / 2,2^{k+j+2}\right)$ ) converge in $C^{i}$ to zero. The first part is just the definition of the WAF end with $l=k+j+2$. The second part instead was discussed in 'elliptic estimates' in section 2.

(II) For every given integer $j \geqslant 0$ there is $\tilde{r}_{j}$ such that for every $\bar{r} \geqslant \tilde{r}_{j}$ the following Harnaktype of estimate holds

$$
1 \leqslant \frac{\sup \left\{u(p) / p \in \mathcal{A}_{\bar{r}}\left(1,2^{k+j+2}\right)\right\}}{\inf \left\{u(p) / p \in \mathcal{A}_{\bar{r}}\left(1,2^{k+j+2}\right)\right\}} \leqslant 2 .
$$

This is deduced from the bound $|\nabla \ln u|_{\bar{r}}^{2} \leqslant R_{\bar{r}} / 2$ (contract the first equation in (1)) and from (I) by the following argument. Let $p_{1}$ and $p_{2}$ be two arbitrary points in $\mathcal{A}_{\bar{r}}\left(1,2^{k+j+2}\right)$ and let $\gamma(s), s \in\left[s_{1}, s_{2}\right]$, be a curve inside an annulus $\Omega_{\bar{r}} \supset \mathcal{A}_{\bar{r}}\left(1,2^{k+j+2}\right)$ joining $p_{1}$ to $p_{2}$ and parametrized by the $g_{\bar{r}}$-arc-length. Then one has

$$
\begin{aligned}
\left|\ln \frac{u\left(p_{2}\right)}{u\left(p_{1}\right)}\right| & =\left|\int_{s_{1}}^{s_{2}} \nabla_{\dot{\gamma}} \ln u \mathrm{~d} s\right| \leqslant \int_{s_{1}}^{s_{2}}|\nabla \ln u|_{\bar{r}} \mathrm{~d} s \\
& \leqslant\left(\sup _{p \in \Omega_{\bar{r}}} \sqrt{\frac{R_{\bar{r}}(p)}{2}}\right) \operatorname{length}_{\bar{r}}(\gamma) .
\end{aligned}
$$

By (I) there is $\tilde{r}_{j}$ such that for any $\bar{r} \geqslant \tilde{r}_{j}$, there is an annulus $\Omega_{\bar{r}} \supset \mathcal{A}_{\bar{r}}\left(1,2^{k+j+2}\right)$ with $\left(\Omega_{\bar{r}}, g_{\bar{r}}\right)$ sufficiently close to $\left(\mathcal{A}_{\mathbb{R}^{3}}\left(1,2^{k+j+2}\right), g_{\mathbb{R}^{3}}\right)$ in $C^{4}$ that any two points in $\mathcal{A}_{\bar{r}}\left(1,2^{k+j+2}\right)$ can be joined through a curve in it of $g_{\bar{r}}$-length less or equal than $2\left(2 \pi+2^{k+j+2}\right.$ ) (by a coarse estimation). By (37), if $\tilde{r}_{j}$ is big enough then

$$
\sup \left\{R_{\bar{r}}(p): p \in \mathcal{A}_{\bar{r}}\left(1,2^{k+j+2}\right)\right\} \leqslant \frac{\ln 2}{2\left(2 \pi+2^{k+j+2}\right)} .
$$

From this and (39) we obtain that for any $p_{1}$ and $p_{2}$ in $\mathcal{A}_{\bar{r}}\left(1 / 2,2^{k+j}\right)$ we have $\left|\ln u\left(p_{2}\right) / u\left(p_{1}\right)\right| \leqslant \ln 2$. The equation (38) then follows.

(III) For any $\bar{r} \geqslant \tilde{r}_{j}$, with $\tilde{r}_{j}$ as in (II), and for any $p \in \mathcal{A}_{\bar{r}}\left(1,2^{k+j+2}\right)$ we have

$$
\left.\frac{1}{2} \chi_{\bar{r}}(p)\right|_{\bar{r}} ^{2} \leqslant \frac{R(p)}{\bar{R}(\bar{r})} \leqslant 2\left|\chi_{\bar{r}}(p)\right|_{\bar{r}}^{2}
$$

where $\chi_{\bar{r}}$ is the following scaling of $\chi=2(u d u+i \omega)=d \mathcal{E}$

$$
\chi_{\bar{r}}:=\frac{\chi}{\overline{\left.\bar{\chi}\right|_{\bar{r}}}}, \quad \text { with } \quad \overline{|\chi|}_{\bar{r}}:=\sup \left\{|\chi(p)|_{\bar{r}}: p \in S(\bar{r})\right\} .
$$

Moreover if $\bar{r} \geqslant \max \left\{\tilde{r}_{j}, 2 / \varepsilon-1\right\}$ then

$$
\left|\chi_{\bar{r}}(p)\right|_{\bar{r}}^{2} \leqslant 2\left(\frac{1}{d_{\bar{r}}(p)}\right)^{\alpha}
$$

for all $p \in \mathcal{A}_{\bar{r}}\left(1,2^{k+j+2}\right)$ and where $\alpha=1=2 \varepsilon$. 
To see the first inequality in (40) operate as follows

$$
\begin{aligned}
\frac{R(p)}{\bar{R}(\bar{r})} & =\frac{\frac{|\chi(p)|^{2}}{2 u^{4}(p)}}{\sup _{\bar{p} \in S(\bar{r})} \frac{|\chi(\bar{p})|^{2}}{2 u^{4}(\bar{p})}}=\frac{|\chi(p)|^{2}}{\sup _{\bar{p} \in S(\bar{r})}|\chi(\bar{p})|^{2} \frac{u^{4}(p)}{u^{4}(\bar{p})}} \geqslant \frac{1}{2} \frac{|\chi(p)|^{2}}{\sup _{\bar{p} \in S(\bar{r})}|\chi(\bar{p})|^{2}} \\
& =\frac{1}{2} \frac{|\chi(p)|_{\bar{r}}^{2}}{\overline{|\chi|_{\bar{r}}^{2}(\bar{r})}}=\frac{1}{2}\left|\chi_{\bar{r}}(p)\right|_{\bar{r}}^{2},
\end{aligned}
$$

where in the third step we use $u(p) / u(\bar{p}) \leqslant 2$ by (38). To obtain the second inequality in (40) instead use in the third step that $u(p) / u(\bar{p}) \geqslant 1 / 2$ by (38) too. The inequality (41) is the consequence of combining (26) and (40).

With (I), (II) and (III) at hand we are in a better position to explain the strategy of proof of the proposition. The idea is to use the Ernst equation in the form (4) to show that there is a constant $\hat{c}$ independent of $k$ and a $r_{k} \geqslant \tilde{r}_{0}$ (here $\tilde{r}_{0}$ is $\tilde{r}_{j}$ with $j=0$ ) such that for every $\bar{r} \geqslant r_{k}$ and $p \in \mathcal{A}_{\bar{r}}\left[2,2^{k+1}\right]$ we have

$$
\left|\chi_{\bar{r}}(p)\right|_{\bar{r}}^{2} \leqslant \frac{\hat{c}}{2}\left(\frac{\bar{r}}{d(p)}\right)^{4} .
$$

Together with (40), this would imply that for every $\bar{r} \geqslant r_{k}$ and $p \in \mathcal{A}_{\bar{r}}\left[2,2^{k+1}\right]$ the inequality

$$
\frac{R(p)}{\bar{R}(\bar{r})} \leqslant \hat{c}\left(\frac{\bar{r}}{d(p)}\right)^{4},
$$

must hold. Letting $c:=\max \{\hat{c}, 16\}$, then, by (26), we would also have

$$
\frac{R(p)}{\bar{R}(\bar{r})} \leqslant 1 \leqslant c\left(\frac{\bar{r}}{d(p)}\right)^{4},
$$

for every $p \in \mathcal{A}_{\bar{r}}[1,2]$. Thus, $R(p) / \bar{R}(\bar{r}) \leqslant c(\bar{r} / d(p))^{4}$ would hold for every $p \in \mathcal{A}_{\bar{r}}\left[1,2^{k}\right]$. Hence, if $\bar{r} \leqslant \hat{r} \leqslant 2^{k} \bar{r}$ we would have $\bar{R}(\hat{r}) \leqslant c(\bar{r} / \hat{r})^{4} \bar{R}(\bar{r})$ as wished.

We move now to prove (43). In the two equations of (4) divide $\chi$ by $\mid \bar{\chi} \overline{\mid}_{\bar{r}}$ (which amounts to make $\chi \rightarrow \chi_{\bar{r}}$ ) and then in the first equation of (4) multiply both sides by $\bar{r}^{2}$ (which amounts to make $g \rightarrow g_{\bar{r}}$ ). In this way one obtains the equivalent system

$$
\left\{\begin{array}{l}
\operatorname{div}_{\bar{r}} \chi_{\bar{r}}=\left\langle\zeta, \chi_{\bar{r}}\right\rangle_{\bar{r}}, \\
\mathrm{~d} \chi_{\bar{r}}=0,
\end{array}\right.
$$

where, recall, $\zeta=2(u \nabla u+\omega i) / u^{2}$. To deduce (43) we will think (44) as a linear elliptic system in the variable $\chi_{\bar{r}}$ and we will consider $\zeta$ as a coefficient.

From (I) we deduce that for any $\bar{r} \geqslant \breve{r}_{j}$, with $\breve{r}_{j}>0$ sufficiently large, there is around every $p \in \mathcal{A}_{\bar{r}}\left[2,2^{k+j+1}\right]$ an harmonic coordinate system $\left\{x=\left(x^{1}, x^{2}, x^{3}\right):|x| \leqslant I_{1}\right\}$, $p=(0,0,0)$, where the system (44) is written in the form 


$$
a^{i j} \partial_{i} \partial_{j} \phi_{\bar{r}}+b^{i} \partial_{i} \phi_{\bar{r}}=0, \quad \partial_{i} \phi_{\bar{r}}=\chi_{\bar{r}}\left(\partial_{i}\right),
$$

with the coefficients $a^{i j}$ uniformly elliptic and uniformly bounded (i.e. by $\bar{r}$-independent bounds) in $C^{4}$ and the coefficients $b^{i}$ uniformly bounded in $C^{2}$ (of course more is known but these bounds are enough). On the other hand from (41) and recalling that we find $\phi_{\bar{r}}$ from $d \phi_{\bar{r}}=\chi_{\bar{r}}$, we deduce that $\phi_{\bar{r}}$ is also uniformly bounded in $C^{1}$ if $\phi_{\bar{r}}$ is set to be zero at $(0,0,0)$. We can then rely on standard interior elliptic estimates over every one of such coordinate systems to conclude that there is a constant $c^{*}>0$ such that for any $\bar{r} \geqslant \breve{r}_{j}$ with $\breve{r}_{j}$ sufficiently large, the $C_{g_{\bar{r}}}^{2}$-norm of $\chi_{\bar{r}}\left(=d \phi_{\bar{r}}\right)$ over $\mathcal{A}_{\bar{r}}\left[2,2^{k+j+1}\right]$ is bounded by $c^{*}$.

From these uniform $C_{g_{r}}^{2}$-bounds for $\chi_{\bar{F}}$ and (I) the following fourth fact is just the result of a standard limit.

(IV) Given $j \geqslant 0$ and a divergent sequence $\left\{\bar{r}_{m}\right\}$, there is a subsequence (indexed again by m) such that, the forms $\chi_{\overline{\bar{T}}_{m}}$ over $\mathcal{A}_{\overline{\bar{r}}_{m}}\left[2,2^{k+j+1}\right]$ converge in $C^{1}$ to a form $\psi$ on $\mathcal{A}_{\mathbb{R}^{3}}\left(2,2^{j+k+1}\right]$ solution of

$$
\left\{\begin{array}{l}
\operatorname{div} \psi=0, \\
\mathrm{~d} \psi=0
\end{array}\right.
$$

and satisfying that,

(a) $|\psi(x)| \leqslant 2 /|x|^{\alpha}$ for every $x$ in $\mathcal{A}_{\mathbb{R}^{3}}\left[2,2^{k+j+1}\right]$, where $\alpha=1-2 \varepsilon$, and that,

(b) the $C^{1}$-norm of $\psi$ over $\mathcal{A}_{\mathbb{R}^{3}}[2,4]$ is bounded by $c^{*}$, where $c^{*}$ is the constant defined before.

The limit form $\psi$ in (IV) then satisfies the hypothesis of proposition 4.5. Therefore proposition 4.5 provides us with a constant $\hat{c}\left(c^{*}\right)$ that is the one we will use now to show (43). Recall that the purpose is to show that there is a constant $\hat{c}$ independent of $k$ and $r_{k}>0$ such that for every $\bar{r} \geqslant r_{k}$ and $p \in \mathcal{A}_{\bar{r}}\left[2,2^{k+1}\right]$ the equation (43) holds. Having chosen $\hat{c}$ as we did, the existence of $r_{k}$ is shown by contradiction. We do that in what follows. Suppose then that there is a divergent sequence $\bar{r}_{m} \rightarrow \infty$ and a sequence of points $p_{m} \in \mathcal{A}_{\bar{r}_{m}}\left[2,2^{k+1}\right]$ such that

$$
\left|\chi_{\bar{r}_{m}}\left(p_{m}\right)\right|_{\bar{r}_{m}}^{2}>\frac{\hat{c}}{2}\left(\frac{\bar{r}_{m}}{d\left(p_{m}\right)}\right)^{4}=\frac{\hat{c}}{2 d_{\bar{r}_{m}}^{4}\left(p_{m}\right)} .
$$

By (IV) with $j=1$, there is a subsequence (indexed again by $m$ ) such that the forms $\chi_{\bar{F}_{m}}$ over $\mathcal{A}_{\bar{r}_{m}}\left[2,2^{k+2}\right]$ converge in $C^{1}$ to a form $\psi$ on $\mathcal{A}_{\mathbb{R}^{3}}\left[2,2^{k+2}\right]$, solution of (45), satisfying (a), (b) and for which, in addition, there is a point $x_{1} \in \mathcal{A}_{\mathbb{R}^{3}}\left[2,2^{k+1}\right]$ with

$$
\left|\psi\left(x_{1}\right)\right|^{2} \geqslant \frac{\hat{c}}{2\left|x_{1}\right|^{4}} .
$$


To this subsequence one can again apply (IV) with $j=2$, to conclude that there is again a subsequence of it (indexed again by $m$ ) such that the forms $\chi_{\bar{r}_{m}}$ over $\mathcal{A}_{\bar{r}_{m}}\left[2,2^{k+3}\right]$ converge in $C^{2}$ to a form $\psi$ on $\mathcal{A}_{\mathbb{R}^{3}}\left[2,2^{k+3}\right]$ solution of (45), satisfying (a), (b) and for which, in addition, there is a point $x_{2} \in \mathcal{A}_{\mathbb{R}^{3}}\left[2,2^{k+1}\right]$ with

$$
\left|\psi\left(x_{2}\right)\right|^{2} \geqslant \frac{\hat{c}}{2\left|x_{2}\right|^{4}} \text {. }
$$

One can continue applying (IV) with $j=3,4, \ldots$ and then taking a diagonal sequence to conclude that there is a form $\psi$ on $\mathbb{R}^{3} \backslash B_{\mathbb{R}^{3}}(o, 2)$ solution of (45), satisfying (a), (b) and for which, in addition, there is a point $x_{\infty} \in \mathcal{A}_{\mathbb{R}^{3}}\left[2,2^{k+1}\right]$ with

$$
\left|\psi\left(x_{\infty}\right)\right|^{2} \geqslant \frac{\hat{c}}{2\left|x_{\infty}\right|^{4}},
$$

which is not possible because of how the constant $\hat{c}$ was defined.

At this point the proof of asymptotic flatness and Schwarzschildian fall off is direct, as observed by Kennefick and Ó Murchadha [4], once the curvature enjoys a $1 / d^{4-\varepsilon}$ decay then it must forcefully enjoy a $1 / d^{4}$ decay and the metric must have Schwarzschildian fall off. For completeness we give the main elements of the construction below.

Proposition 4.6. Let $(E ; g, \omega, u)$ be a WAF end. Then, there is a coordinate system $\left\{x=\left(x^{1}, x^{2}, x^{3}\right)\right\}$ covering $E$ up to a compact set such that

$$
\begin{gathered}
\left|\delta_{i j}-g_{i j}\right| \leqslant K /|x|^{2}, \quad\left|\partial_{k} g_{i j}\right| \leqslant K /|x|^{3}, \quad\left|\partial_{m} \partial_{k} g_{i j}\right| \leqslant K /|x|^{4}, \quad \text { and, } \\
\left|\partial_{i} u\right|+\left|\omega_{i}\right| \leqslant K /|x|^{2},
\end{gathered}
$$

plus further progressive power-law decay for the norms of the multiple $\partial$-derivatives of $\tilde{g}_{i j}, u$ and $\omega_{i}$, where here $K$ is a positive constant and $|x|$ is the norm of $x=\left(x^{1}, x^{2}, x^{3}\right)$ as a vector in $\mathbb{R}^{3}$.

Note that $g_{i j}$ decays faster than $\tilde{g}_{i j}=u^{2} g_{i j}$ according to definition 1.4. It is indeed the factor $u^{2}$ that causes a slower decay for $\tilde{g}_{i j}$.

Proof. From (1) we have $|(\nabla \ln u)(p)|^{2} \leqslant R(p) / 2 \leqslant c_{\eta} / 2 d(p)^{4-\eta}$. Then, scaling $u$ and $\omega$ if necessary, deduce (integrating along paths) that $\ln u$ goes to zero at infinity and furthermore that $|\ln u(p)| \leqslant c^{\prime}{ }_{\eta} / d(p)^{1-\eta / 2}$. Similarly, if $\phi$ is a potential for $\omega$, then by (1) we have $|\nabla \phi|^{2} \leqslant u^{4} R / 2 \leqslant \bar{c}_{\eta} / d^{4-\eta}$. Therefore by adding a constant if necessary the potential $\phi$ goes to zero at infinity and we have $|\phi(p)| \leqslant \bar{c}^{\prime}{ }_{\eta} / d(p)^{1-\eta / 2}$. Summarizing,

$$
|\ln u(p)|+|\phi(p)| \leqslant \frac{c}{d^{1-\eta / 2}(p)}, \quad \text { and },
$$




$$
|(\nabla \ln u)(p)|+|(\nabla \phi)(p)| \leqslant \frac{c}{d^{1-\eta / 2}(p)} .
$$

We will use below the following claim: Let $1>\eta \geqslant 0$ (including the possibility $\eta=0$ ). If (48) holds then,

$$
|\operatorname{Ric}(p)| \leqslant \frac{b_{1}}{d^{4-\eta}(p)}, \quad \text { and } \quad|\nabla \operatorname{Ric}(p)| \leqslant \frac{b_{1}}{d^{5-\eta}(p)},
$$

and there is an harmonic coordinate system $\left\{x=\left(x^{1}, x^{2}, x^{3}\right)\right\}$ on which we have

$$
\begin{aligned}
& \left|\delta_{i j}-g_{i j}\right| \leqslant \frac{b_{2}}{|x|^{2-\eta}}, \quad\left|\partial_{k} g_{i j}\right| \leqslant \frac{b_{2}}{|x|^{3-\eta}}, \quad\left|\partial_{l} \partial_{k} g_{i j}\right| \leqslant \frac{b_{2}}{|x|^{4-\eta}}, \\
& \left|\partial_{i} \ln u\right| \leqslant \frac{b_{2}}{|x|^{2-\eta / 2}}, \quad \text { and } \quad\left|\partial_{i} \phi\right| \leqslant \frac{b_{2}}{|x|^{2-\eta / 2}} .
\end{aligned}
$$

Let us postpone the proof of the claim until later and use it now, say with $\eta=1 / 2$. Then, from (1), $\ln u$ and $\phi$ satisfy the equations

$$
\Delta \ln u=f_{1}=O\left(\frac{1}{|x|^{7 / 2}}\right), \quad \text { and } \quad \Delta \phi=f_{2}=O\left(\frac{1}{|x|^{7 / 2}}\right),
$$

where we are thinking $f_{1}$ and $f_{2}$ as sources. With this fast decay of the sources we get for both, $\ln u$ and $\phi$, a decay $O(1 /|x|)$, namely as in (48) with $\eta=0 .{ }^{9}$ Then, (51) and (52) with $\eta=0$ are just (46) and (47) respectively. The further progressive power-law decay is achieved by a standard elliptic bootstrap of decay and is unnecessary to include here.

To finish the proof we need to explain how to prove the claim. From (48) and for any $p \in \mathcal{A}_{r}(1 / 2,2)$ we have $|\phi(p)| \leqslant c / d(p)^{1-\eta / 2}=c(r / d(p))^{1-\eta / 2}(1 / r)^{1-\eta / 2} \leqslant 2 c / r^{1-\eta / 2}$ and $|(\nabla \phi)(p)|_{r} \leqslant c / d(p)^{2-\eta / 2}(1 / r)^{2-\eta / 2}=c(r / d(p))^{2-\eta / 2}(1 / r)^{2-\eta / 2} \leqslant 4 c / r^{2-\eta / 2}$. Similar bounds are obtained for $\ln u$. Summarizing, all over $\mathcal{A}_{r}(1 / 2,2)$ we have the uniform bounds

$$
|\ln u|+|\phi| \leqslant 4 c / r^{1-\eta / 2}, \quad \text { and } \quad|\nabla \ln u|_{r}+|\nabla \phi|_{r} \leqslant 8 c / r^{1-\eta / 2} .
$$

We will use them to obtain interior elliptic estimates on the smaller annuli $\mathcal{A}_{r}(4 / 5,5 / 4)$. Recall that when $r \rightarrow \infty$ the annulus $\left(\mathcal{A}_{r}(1 / 2,2), g_{r}\right)$ converges to $\left(\mathcal{A}_{\mathbb{R}^{3}}(1 / 2,2), g_{\mathbb{R}^{3}}\right)$ in $C^{i}$ for every $i \geqslant 2$ and due to this we do not need to worry about the constants involved in Sobolev embeddings or elliptic estimates if $r$ is sufficiently large. Scaling (1) gives on $\mathcal{A}_{r}(1 / 2,2)$

$$
\Delta_{r} \ln u=-2 \frac{|\nabla \phi|_{r}^{2}}{u^{4}}=f_{1, r}, \quad \text { and } \quad \Delta_{r} \phi=4\langle\nabla \ln u, \nabla \phi\rangle_{r}=f_{2, r}
$$

where we will think of $f_{r, 1}$ and $f_{r, 2}$ as sources. Then, $L^{p}$ interior elliptic estimates [3] give $\|\ln u\|_{H_{g_{r}}^{2,4}\left(\mathcal{A}_{r}(3 / 5,5 / 3)\right)} \leqslant c_{1}\left(\left\|f_{1, r}\right\|_{L_{g_{r}}^{4}\left(\mathcal{A}_{r}(1 / 2,2)\right)}+\|\ln u\|_{L_{g_{r}}^{4}\left(\mathcal{A}_{r}(1 / 2,2)\right)}\right) \leqslant \frac{c_{2}}{r^{1-\eta / 2}}$,

where to obtain the last inequality use (53) (assume $r>1$ ). Sobolev embeddings then give $\|\ln u\|_{C_{g_{r}}^{1, \beta}\left(\mathcal{A}_{r}(3 / 5,5 / 3)\right)} \leqslant c_{3} / r^{1-\eta / 2}$. In the same way we obtain

9 The reader can check that ||$x|-d(x)| \leqslant$ constant. 
$\|\phi\|_{C_{g_{r}}^{1, \beta}\left(\mathcal{A}_{r}(3 / 5,5 / 3)\right)} \leqslant c_{4} / r^{1-\eta / 2}$. Use these bounds to get $C^{1, \beta}$ bounds for the sources $f_{1, r}$ and $f_{2, r}$ and from them and Schauder estimates [3] get the bound $\|\ln u\|_{C_{g_{r}}^{2, \beta}\left(\mathcal{A}_{r}(4 / 5,5 / 4)\right)} \leqslant c_{5} / r^{1-\eta / 2}$ and $\|\phi\|_{C_{g_{r}}^{2, \beta}\left(\mathcal{A}_{r}(4 / 5,5 / 4)\right)} \leqslant c_{5} / r^{1-\eta / 2}$. In particular if $r=d(p)$ and by undoing the scaling we have $|(\nabla \nabla \ln u)(p)| \leqslant c_{5} / r^{3-\eta / 2}=c_{5} / d(p)^{3-\eta / 2}$ and $|(\nabla \nabla \phi)(p)| \leqslant c_{5} / r^{3-\eta / 2}=c_{5} / d(p)^{3-\eta / 2}$. Use these estimates and (49) in (1) to arrive easily at (50). Finally, as shown in [2] (theorem on page 314, with $\eta 9$ equal to $2-\eta$ here; see also remark 1) the cubic volume growth and the curvature decays (50) are enough to guarantee the existence of a coordinate system $\left\{x=\left(x^{1}, x^{2}, x^{3}\right)\right\}$ satisfying (51). The proof of the claim is finished.

\section{References}

[1] Anderson M T 2000 On stationary vacuum solutions to the Einstein equations Ann. Henri Poincaré 1 977-94

[2] Bando S, Kasue A and Nakajima H 1989 On a construction of coordinates at infinity on manifolds with fast curvature decay and maximal volume growth Invent. Math. 97 313-49

[3] Gilbarg D and Trudinger N S 1983 Elliptic Partial Differential Equations of Second Order 2nd edn (Berlin: Springer)

[4] Kennefick D and Murchadha N Ó 1995 Weakly decaying asymptotically flat static and stationary solutions to the Einstein equations Class. Quantum Grav. 12 149-58

[5] Meinel R, Ansorg M, Kleinwächter A, Neugebauer G and Petroff D 2008 Relativistic Figures of Equilibrium (Cambridge: Cambridge University Press)

[6] Ehlers J 1980 Isolated systems in general relativity Ann. New York Acad. Sci. 336 279-94

[7] Persic M, Salucci P and Stel F 1996 The universal rotation curve of spiral galaxies: 1. The dark matter connection Mon. Not. R. Astron. Soc. 28127

[8] Petersen P 2006 Riemannian Geometry (Graduate Texts in Mathematics) vol 171 2nd edn (New York: Springer)

[9] Reris M 2013 On Ricci curvature and volume growth in dimension three J. Differ. Geom. (arXiv: 1212.1317)

[10] Salucci P et al 2007 The universal rotation curve of spiral galaxies. 2. The dark matter distribution out to the virial radius Mon. Not. R. Astron. Soc. $37841-47$

[11] Sofue Y and Rubin V 2001 Rotation curves of spiral galaxies Ann. Rev. Astron. Astrophys. 39 137-74

[12] Stephani H, Kramer D, MacCallum M, Hoenselaers C and Herlt E 2003 Exact solutions of Einstein's field equations Cambridge Monographs on Mathematical Physics 2nd edn (Cambridge: Cambridge University Press)

[13] Binney J and Tremaine S 2008 Galactic Dynamics 2nd edn (New Jersey: Princeton University Press)

[14] Wald R M 1984 General Relativity (Chicago, IL: University of Chicago Press)

[15] Zaritsky D 1998 The mass and extent of the galactic halo The Third Stromlo Symposium: The Galactic Halo vol 165 ed B K Gibson, T S Axelrod and M E Putman p 34 